\title{
Structure of Mycobacterium tuberculosis Phe-tRNA synthetase reveals ligand binding and two-stage tRNA recognition
}

\author{
Karolina Michalska \\ UChicago/ANL \\ Robert Jedrzejczak \\ UChicago/ANL \\ Jacek Wower \\ Auburn University \\ Changsoo Chang \\ UChicago/ANL \\ Beatriz Baragaña \\ University of Dundee \\ Ian H. Gilbert \\ University of Dundee \\ Barbara Forte \\ University of Dundee \\ Andrzej Joachimiak ( $\sim$ andrzejj@anl.gov ) \\ UChicago/ANL
}

\section{Research Article}

Keywords: Phe-tRNA synthetase, PheRS, FRS, tRNA binding, adenylation, aminoacylation, crystal structure, enzyme inhibitor, catalysis, tuberculosis, Mycobacterium tuberculosis,

Posted Date: October 9th, 2020

DOl: https://doi.org/10.21203/rs.3.rs-89886/v1

License: (c) (i) This work is licensed under a Creative Commons Attribution 4.0 International License.

Read Full License 


\title{
Structure of Mycobacterium tuberculosis Phe-tRNA synthetase reveals ligand binding and two-stage tRNA recognition
}

\author{
Karolina Michalska ${ }^{1,2}$, Robert Jedrzejczak ${ }^{1,2}$, Jacek Wower ${ }^{3}$, Changsoo Chang ${ }^{1,2}$, Beatriz \\ Baragaña ${ }^{4}$, Ian H. Gilbert ${ }^{4}$, Barbara Forte ${ }^{4}$, and Andrzej Joachimiak ${ }^{1,2,5 \rrbracket}$ \\ ${ }^{I}$ Center for Structural Genomics of Infectious Diseases, Consortium for Advanced Science and \\ Engineering, University of Chicago, Chicago, Illinois 60667, USA \\ ${ }^{2}$ Structural Biology Center, X-ray Science Division, Argonne National Laboratory, Lemont, \\ Illinois 60439, USA \\ ${ }^{3}$ Auburn University, Department of Animal Sciences, Auburn, AL 36849, USA \\ ${ }^{4}$ Drug Discovery Unit, Wellcome Centre for Anti-Infectives Research, Division of Biological \\ Chemistry and Drug Discovery, University of Dundee, Dundee, DDI 5EH, UK \\ ${ }^{5}$ Department of Biochemistry and Molecular Biology, University of Chicago, Chicago, IL, \\ 60367, USA
}

"Correspondence should be addressed to: Andrzej Joachimiak, Structural Biology Center, X-ray Science Division Argonne National Laboratory, Lemont, Illinois 60439, USA

Phone: 630-252-3926; fax: 630-252-6126; e-mail: andrzejj@anl.gov

Running Title: Complexes of Phe-tRNA synthetase from Mycobacterium tuberculosis

Keywords: Phe-tRNA synthetase, PheRS, FRS, tRNA binding, adenylation, aminoacylation, crystal structure, enzyme inhibitor, catalysis, tuberculosis, Mycobacterium tuberculosis, 


\begin{abstract}
Tuberculosis, caused by Mycobacterium tuberculosis, responsible for $\sim 1.5$ million fatalities in 2018 , is the deadliest infectious disease. Global spread of multidrug resistant TB is a public health threat. Aminoacyl-tRNA synthetases are plausible candidates as potential targets because they play pivotal roles in translating the DNA code into protein sequence by attaching specific amino acid to their cognate tRNAs. One of the best characterized synthetases is specific for L-Phe and tRNA ${ }^{\text {Phe }}$. Here we report structures of $M$. tuberculosis Phe-tRNA synthetase complexed with precursor tRNA and either phenylalanine or a phenylalanine adenylate analog, 5'-O-(N-phenylalanyl)sulfamoyl-adenosine. Crystallographic models for the first time reveal two modes of interaction with tRNA: an initial recognition via the anticodon loop and stem only and productive binding with interaction of the 3 ' end of tRNA ${ }^{\text {Phe }}$ with the adenylate site. We biochemically characterize the enzyme and provide the highest resolution, most complete view of the Phe-tRNA synthetase/tRNA ${ }^{\text {Phe }}$ system to date.
\end{abstract}




\section{Introduction}

With 1.5 million people killed in 2018, Mycobacterium tuberculosis, the etiological agent of the tuberculosis (TB) ${ }^{1}$ remains the deadliest pathogen worldwide ${ }^{1}$ and the leading cause of morbidity and mortality among HIV-infected patients. It is estimated that one third of the world's population is at risk of developing active TB disease, especially in low and middle income countries. Extensively drug resistant TB (XDR-TB) and multidrug-resistant tuberculosis (MDR-TB) is a growing global public health threat ${ }^{2}$. The existing TB multi-drug treatments are long, particularly for XDR-TB and MDR-TB infections. Discovery of new drugs with novel mechanisms of action are critical to treat infections with existing drug resistant strains and to shorten TB treatment ${ }^{3}$.

Protein translation is an essential processes and the target of Linezolid an approved drug for TB treatment and several other compounds in clinical trials for $\mathrm{TB}{ }^{4,5}$. One of the crucial steps in protein synthesis involves the enzymatic attachment of a specific amino acid to its cognate tRNA ${ }^{6}$, carried out by aminoacyl-tRNA synthetases (aaRS). In addition, some aaRSs deploy proofreading mechanisms to ensure correct pairing. The synthesis of aminoacyl-tRNA is accomplished by the 2' or 3'-esterification of tRNA with an appropriate amino acid in a two-step reaction. First, the aminoacyl-adenylate is formed by reaction between ATP and an amino acid, sometimes in a tRNAdependent manner ${ }^{7}$, followed by ligation to the adenosine of tRNA.

FRS, which is specific for L-phenylalanine and $\mathrm{tRNA}^{\text {Phe }}$, has been studied extensively biochemically, structurally and computationally for the past 50 years ${ }^{8-11}$. Several FRS structures at moderate resolution are available in Protein Data Bank (PDB) from bacteria, archaea and eukaryotes (Table 1). FRS belongs to the class IIC aaRS but it is considered an outlier and shows similarity to class IID enzymes SRS and ancient GRS and ARS ${ }^{6}$. Typically, class II aaRSs aminoacylate tRNA on 3'OH of ribose, but FRS does so on 2'OH. The bacterial, archaeal and human cytoplasmic enzymes consist of $\alpha$ (PheS) and $\beta$ subunits (PheT) that via domain swapping form highly intertwined $(\alpha \beta)_{2}$ heterotetramers ${ }^{10,12-16}$. The $\alpha$ subunit recognizes L-Phe and 3' terminal adenosine of tRNA and carries out key catalytic activities ${ }^{17}$. The $\beta$ subunit recognizes tRNA ${ }^{\text {Phe }}$ and provides editing function assuring that the L-Phe is attached to tRNA ${ }^{\text {Phe } 18}$.

\footnotetext{
${ }^{1}$ Abbreviations used: aaRS, aminoacyl-tRNA synthetase; F-AMS, 5'-O-(N-phenylalanyl)sulfamoyl-adenosine; FRS, phenylalanyl-tRNA synthetase; hmFRS, human mitochondrial FRS; hcFRS, human cytosolic FRS; TB, tuberculosis;
} 
As essential enzymes, aaRS have been for a long time considered as drug targets ${ }^{19,20}$. For TB, an inhibitor of LRS has recently completed Phase 1 clinical trials ${ }^{4}$. FRS has been recognized as a highpriority antimicrobial drug target ${ }^{18,20}$ and several inhibitors have been reported. Phenyl-thiazolylureasulfonamides are selective and potent inhibitors of both Gram-negative and Gram-positive bacterial FRS orthologs ${ }^{8}$. Other bacterial FRS inhibitors include ethanolamine derivatives for Staphylococcus aureus $^{21}$, gossypol for Pseudomonas aeruginosa and Streptococcus pneumoniae 22 and some natural products for $M t \mathrm{FRS}^{23}$. However, no FRS inhibitor has reached advanced therapeutic development stages.

Thus far, M. tuberculosis FRS has not been characterized, hampering structure-based drug discovery approaches. Here, we address this major knowledge gap and present four crystal structures of $M t$ FRS in complex with a specific tRNA ${ }^{\text {Phe }}$ precursor $\left(M t \mathrm{RNA}^{\text {Phe }}\right)$. For the first time we show tRNA recognition and binding occurring in two stages. The "initial tRNA recognition" state was determined with either bound L-Phe (in two crystal forms), or with an inhibitor - a nonhydrolyzable phenylalanine adenylate analog 5'-O-(N-phenylalanyl)sulfamoyl-adenosine (F-AMS). The structure of productive tRNA aminoacylation state that is predisposed for acceptance of an activated amino acid was determined in complex with F-AMS. These structures offer the most complete picture of the FRS-tRNA ${ }^{\text {Phe }}$ complex to date and combined with biochemical characterization of the enzyme and F-AMS binding provide the bases for rational structure-based drug design of selective inhibitors of $M t \mathrm{FRS}$.

\section{Results and Discussion}

\section{tRNA ${ }^{\text {Phe }}$ and F-AMS binding}

To investigate structure-function relationship of $M t \mathrm{FRS}$, we have co-expressed PheS $(\alpha)$ and PheT $(\beta)$ subunits of $M t$ FRS and purified the functional heterotetrameric complex. In addition, we have prepared unmodified $M$. tuberculosis tRNA ${ }^{\text {Phe }}$ precursor specific for the 5'UUC codon - isoacceptor $M t t_{\text {RNA }}^{\text {Phe }}$ with the GAA3' anticodon. This synthetic $M t t_{\text {RNA }}^{\text {Phe }}$ lacks post-transcriptional modifications. The actual nature of genus-specific modifications is unknown but in other organisms tRNA $^{\text {Phe }}$ has been shown to carry many variations: $\mathrm{m}^{2} \mathrm{G}, \mathrm{D}, \mathrm{Cm}^{3}, \mathrm{Gm}^{3}, \mathrm{Cm}, \mathrm{Gm}, 2$-thiomethyl-N6(delta2-isopentenyl)A, $\mathrm{m}^{1} \mathrm{G}, \mathrm{m}^{5} \mathrm{C}, \Psi, \mathrm{m}^{1} \mathrm{C}, 3$-(3-carboxypropyl) $\mathrm{U}, \mathrm{T}, \mathrm{m}^{1} \mathrm{~A}$ and in eukaryotic tRNAs also large wybutosine ${ }^{24}$. The majority of these variations have little effect on aminoacylation by 
aaRSs but are important for codon - anticodon interactions, translational rates, reading frame maintenance, co-translational folding dynamics and other cellular processes ${ }^{25,26}$.

To confirm enzymatic activity of the protein, we have independently tested both reaction steps, L-Phe adenylation and tRNA aminoacylation, with three substrates: synthetic $M t$ RNA ${ }^{\text {Phe }}$ precursor as well as $E$. coli and yeast $\mathrm{tRNA}^{\text {Phe }}$. Unmodified $M t \mathrm{tRNA}^{\text {Phe }}$ can be almost completely ( $\left.>99 \%\right)$ charged by $M t$ FRS with L-Phe (Fig. 1). MtFRS can also aminoacylate mature tRNA ${ }^{\text {Phe }}$ molecules isolated from E. coli and yeasts (Fig. 1). $M t$ FRS, like all known FRS, is able to activate its amino acid substrate in the absence of tRNA (Fig. 1A-1C), though the addition of tRNA stimulates this reaction.

Aminoacyl-sulfamoyl adenosine have been previously been reported as inhibitors of bacterial aaRS ${ }^{27-29}$. Here, we report strong inhibition of $M t$ FRS by the non-hydrolyzable phenylalanine adenylate analog, 5'-O-[N-(phenylalanine)sulfamoyl] adenosine (F-AMS). Earlier studies have demonstrated that aminoacyl-sulfamoyl-adenosines can efficiently inhibit E. coli $\mathrm{ERS}\left(\mathrm{K}_{\mathrm{i}} 2.8 \mathrm{nM}\right)^{27}$, murine liver ERS $\left.\left(\mathrm{K}_{\mathrm{i}} \sim 70 \mathrm{nM}\right)^{27}\right)$, E. coli QRS $\left(\mathrm{K}_{\mathrm{i}} 1.3 \mu \mathrm{M}\right)$, S. aureus IRS $\left(\mathrm{IC}_{50} 4 \mathrm{nM}\right)^{29}, \mathrm{RRS}\left(\mathrm{IC}_{50}\right.$ $4.5 \mathrm{nM}), \mathrm{HRS}\left(\mathrm{IC}_{50} 130 \mathrm{nM}\right)$ and TRS (IC $\left.5015 \mathrm{nM}\right)^{28}$. F-AMS acts as a competitive inhibitor with a $\mathrm{K}_{\mathrm{i}}$ of $33 \mathrm{nM}$ and $\mathrm{IC}_{50}$ value of approximately $36 \mathrm{nM}$ (Fig. 2).

\section{Overall structure}

To understand structural basis of aminoacylation reaction and tRNA recognition, we have crystallized $M t$ FRS with $M t t_{\text {RNA }}^{\text {Phe }}$ and either L-Phe or F-AMS. Four structures have been determined - two representing different crystal forms of the $M t \mathrm{tRNA}{ }^{\text {Phe }} / \mathrm{L}-\mathrm{Phe}$ complex $(M t \mathrm{FRSt} / \mathrm{F} 1$, and $M t \mathrm{FRSt} / \mathrm{F} 2$ ) and two corresponding to different forms of the $M t$ tRNA ${ }^{\text {Phe }} / \mathrm{F}$-AMS complex (MtFRSt/F-AMS1 and MtFRSt/F-AMS2) (Fig. 3, Table 2). The former three structures illustrate the initial tRNA recognition state, where the enzyme binds primarily to the anticodon region, with only partial contacts with D- and T-loops of tRNA. In these structures the electron density for the acceptor end of tRNA is poorly defined and cannot be modeled. The MtFRSt/F-AMS2 structure corresponds to the enzymatically productive tRNA binding, where the complex of $M t$ FRS with tRNA ${ }^{\text {Phe }}$ is predisposed for acceptance of the activated amino acid. $M t \mathrm{FRSt} / \mathrm{F} 2$ and $M t \mathrm{FRSt} / \mathrm{F}-\mathrm{AMS} 1$ are isomorphous and contain a single $\alpha \beta$ unit in the asymmetric unit (ASU), while $M t \mathrm{FRSt} / \mathrm{F} 1$ and $M t$ FRSt/F-AMS2 have an entire $(\alpha \beta)_{2}$ heterotetramer in the ASU of different unit cells. The crystal lattice or the tRNA binding status do not seem to affect the protein interactions with L-Phe, F-AMS and tRNA ligands. 
The structures of enzyme with specific $M t t R \mathrm{NA}^{\text {Phe }}$ precursor shows the previously described tetrameric assembly (Fig. 3). The electron density for protein, tRNA, L-Phe, F-AMS is excellent and with a few exceptions, as discussed earlier and below, the entire structure can be modeled. The $(\alpha \beta)_{2}$ assembly has a complex shape that can fit into rectangular prism with overall dimensions $70 \AA$ x 110 $\AA$ x $170 \AA$. The individual subunits are composed of several domains, which structurally and functionally make sense only when assembled into highly intertwined heterotetramer of this intricate molecular machine. Each tRNA molecule interacts with all four polypeptide chains.

Our high-resolution structures allow us to improve domain assignment and their boundaries. The smaller $\alpha$ subunit (modeled residues 3 - 341) is very extended $(\sim 130 \AA)$ and has two distinct domains, $\alpha 1$ and $\alpha 2$, connected by a 14-residue linker (Fig. 4, Supplemental Fig. S2). The N-terminal $\alpha 1$ all helical domain binds the tRNA D-loop region and the C-terminal $\alpha 2$ domain performs aminoacylation. The larger $\beta$ subunit (modeled residues 1 - 831) can be subdivided into three globular domains $\beta 1-3$, two of which have subdomains (Fig. 4, Supplemental Fig. S2). $\beta 1$ has four distinct subdomains): $\beta 1 \mathrm{~s} 1, \beta 1 \mathrm{~s} 2$ and $\beta 1 \mathrm{~s} 3$ corresponding to the editing domain that shares fold with $\alpha 2$ catalytic domain (CATH domain 3.30.930.10), and $\beta 1 \mathrm{~s} 4$. The latter, a small $\alpha / \beta$ subdomain together with $\beta 1 \mathrm{~s} 1$ interacts with the $\alpha 2$ aminoacylation domain via direct protein-protein contacts and a conserved magnesium ion. The $\beta 1 \mathrm{~s} 1, \beta 1 \mathrm{~s} 3$ and $\beta 1 \mathrm{~s} 4$ subdomains form a core of $\beta 1$ and $\beta 1 \mathrm{~s} 2$ is on its periphery. $\beta 1 \mathrm{~s} 4$ is followed by a 13 -residue linker connecting to the $\beta 2$ domain, which consists of two subdomains - larger $\beta 2 \mathrm{~s} 1$ and smaller $\beta 2 \mathrm{~s} 2$. $\beta 2$ interacts extensively with aminoacylation domain $\alpha 2$ (surface area $\sim 2,150 \AA^{2}$ ) contributing significantly to the complex interface. $\beta 2$, via a 15-residue linker, leads to the $\mathrm{C}$-terminal domain $\beta 3$ that extends out of the main body of the protein and is responsible for tRNA anticodon binding.

\section{MttRNA ${ }^{\text {Phe }}$ structure}

The complete $M t t_{\mathrm{RNA}}{ }^{\text {Phe }}$ molecules are modeled only in the $M t \mathrm{FRSt} / \mathrm{F}-\mathrm{AMS} 2$ complex, where the only atoms missing from the model correspond to the linkage between U22 and G23. Notably, this structure has been determined at high magnesium concentration and several $\mathrm{Mg}^{2+}$ ions have been identified - some of them are highly hydrated and bind exclusively to the nucleic acid via electrostatic interactions rather than direct metal ion coordination. The complete structure of $M t \mathrm{RNA}^{\mathrm{Phe}}$ is similar to the partial structure of the $M t \mathrm{RNA}^{\text {Phe }}$ in the initial recognition structure (see below) and other structures: the mature yeast equivalent carrying the $\mathrm{Y}$ base in the anticodon region (PDB id 4TNA), 
T. thermophilus precursor tRNA ${ }^{\text {Phe }}$ in complex with FRS (PDB id 2IY5) and unmodified tRNA ${ }^{\text {Phe }}$ from E. coli (PDB id 3L0U). Sequence identity of these tRNAs varies between 45 to $60 \%$ and their superposition indicates that root mean square deviations (RMSD) between them varies between 2.3 to $3.2 \AA$, with the largest differences observed for $M t t \mathrm{RNA}^{\text {Phe }}$ and $T t t \mathrm{RNA}^{\text {Phe }}$. There is one magnesium site in the D-loop region conserved between yeast, E. coli and MttRNA ${ }^{\text {Phe }}$. As expected, the largest differences are in the 3' and 5' ends, anticodon, T- and D-loops. Some of the discrepancies may result from the fact that we compare free tRNA ( $E$. coli and yeast) with tRNA bound to protein $(M$. tuberculosis and T. thermophilus). In fact, anticodon region of these last two tRNAs are more similar to each other. The anticodon loop after the U34 base forms a canonical U-turn stabilized by an internal hydrogen bond of U34 N3 to phosphoryl group of A37 and 2'OH to N7 of A36. This element is conserved in all four structures. In $M t \mathrm{RNA}^{\mathrm{Phe}}$, six consecutive purine bases in the anticodon region (G35 to G40) stack together and form a rigid structure important for tRNA recognition (see below), while E. coli, T. thermophilus and yeast tRNAs have a block of five stacked purine bases. Majority of bases are involved in base-pairing/stacking, but curiously a number of bases project into solvent in different tRNAs and they correspond to this same RNA regions. For example: C17, U20, U48 ( $M$. tuberculosis), U20, U45 (E. coli), dhU17, U47 (yeast), U17, U47 (T. thermophilus). These structural features must be conserved for functional reasons.

\section{Ligand binding}

The active site of FRS adenylation domain of the $\alpha$ subunit contains subpockets responsible for recognition of L-Phe and ATP substrates. Our structures illustrate how L-Phe and its adenylate analog bind to their respective sites (Fig. 5). In the phenylalanine-dedicated subpocket (Fig. 5), $\alpha$ Phe255, $\alpha$ Phe257, $\alpha$ Ala305, $\alpha$ Arg201, and $\alpha$ Thr258 interact with the aromatic ring of the ligand in both $M t$ FRSt/F1 and MtFRSt/F2 structures. $\alpha \operatorname{Arg} 201$ and $\alpha \mathrm{Gln} 215$ form hydrogen bonds with the carboxyl group while the amino group is bonded to $\alpha \mathrm{His} 175$ and, via a water molecule, to $\alpha \mathrm{G} \ln 215$ and $\alpha$ Glu217. The latter residue participates in an extended network of hydrogen bonds which, besides $\alpha \mathrm{Gln} 215$, includes $\alpha$ Ser177 and $\alpha \mathrm{Gln} 180$. The organization of the L-Phe binding subpocket is virtually identical in our two L-Phe complex structures. Some discrepancies are observed in the neighborhood that does not directly participate in the amino acid recognition: the conformations of some side chains facing the adenine binding site differ between the two structures and FRSt/F2 contains $\mathrm{Mg}^{2+}$ ion bound nearby the pocket, interacting with $\alpha \mathrm{Glu} 263$, aGlu279 and a water 
molecule.

An analogous pattern of interaction with phenylalanyl portion of the F-AMS ligand is observed in the $M t \mathrm{FRSt} / \mathrm{F}-\mathrm{AMS} 1$ and $M t \mathrm{FRSt} / \mathrm{F}$-AMS2 complexes. Additional bonds are formed with sulfamoyl group, ribose and adenine moieties. The former interacts with the guanidinium group of $\alpha$ Arg201 and sometimes Glu263 (via a water molecule) while the sugar ring forms hydrogen bonds with $\alpha \mathrm{G} \ln 215$, main chain carbonyl oxygen atom of $\alpha \operatorname{Trp} 280$ and carboxyl group of $\alpha \mathrm{Glu} 279$ (in chain A of $M t \mathrm{FRSt} / \mathrm{F}-\mathrm{AMS} 2)$. The nucleotide base is anchored between $\alpha$ His 209 and $\alpha \mathrm{Phe} 213$ and participates in several hydrogen bonds: (i) N1 with main chain nitrogen atom of $\alpha \mathrm{Thr} 210$, (ii) N3 via a water molecule with main chain of $\alpha \operatorname{Arg} 312$ and $\alpha$ His214, and side chain of $\alpha$ Glu311, (iii) N7 with $\alpha$ Arg201 and $\alpha$ Asp203 via another molecule, and (iv) N6 with main chain carbonyl group of $\alpha$ Thr210 and carboxyl group of $\alpha$ Asp203. Most of these interactions are conserved between the two F-AMS complexes, regardless of the tRNA binding status, with the exception of $\alpha$ Glu279, $\alpha$ Glu263 and N7mediated contact. The adenine subpocket, in the absence of a biological molecule, is occupied by the glycerol molecule.

The conformation of $\alpha 2$ domain is susceptible to the type of ligand, as clear rearrangements are observed when L-Phe and F-AMS complexes are compared. Specifically, in the presence of F-AMS, the loop connecting $\beta 4$ and $\beta 5$ elements from the $\beta 4 \uparrow \beta 5 \downarrow \beta 9 \uparrow \beta 8 \downarrow \beta 7 \uparrow \beta 6 \downarrow$ sheet moves towards the $\beta 8 \downarrow \beta 7 \uparrow \beta 6 \downarrow$ half closing over the nucleotide moiety. This movement enables $\alpha$ His 209 and $\alpha$ Asp203 to approach the F-AMS base ring (Fig. 5). The $\beta 8 \downarrow \beta 7 \uparrow \beta 6 \downarrow$ section, especially the $\beta 7-\beta 8$ hairpin also comes closer to the $\beta 4-\beta 5$ loop.

\section{Initial tRNA ${ }^{\text {Phe }}$ recognition}

In the assembly, each of the tRNA molecules contacts all four subunits through mostly $\alpha 1$ 'and $\beta 3$ ' domains and to a lesser extent $\alpha 2, \beta 1$, and $\beta 2$ (Fig. 3). Remarkably, tRNA recognition is accomplished by one $\alpha \beta$ unit while aminoacylation and editing are completed by the complementary $\alpha^{\prime} \beta$ ' dimer. In the initial recognition state, the anticodon-, D- and T- stem and loop regions are wellordered. On the other hand, the 3' and 5' ends of tRNA are disordered and consequently only 5-70 tRNA bases could be modeled. In these structures, access to the aminoacylation site is blocked by the $3_{10}$-helix region of the $\alpha 2$ domain (residues 152-157, discussed below).

The tRNA selectivity is accomplished by enzyme interacting with the anticodon region of 
tRNA $^{\text {Phe }}$ via anticodon binding domain $\beta 3$ ' (Fig. 6). The rigid 6-purine stack (G35 to G40) seems to be the key structural feature recognized by protein that provides a shallow surface with several specific contact points. The conserved $\beta$ 'Phe780 (FRSs have either phenylalanine or tyrosine in this position) interact via $\pi-\pi$ stacking with the G35 guanine wobble anticodon base, extending the GAAAAG RNA stack. In addition, G35 forms several direct hydrogen bonds. Specifically, $\beta$ 'Asp778 hydrogen bonds to G35 N1 and N2, $\beta$ 'Arg830 to O6 and N7, and $\beta$ 'Gln784 interacts with G35 phosphoryl group or ribose ring oxygen atom (observed in two structures of the initial recognition state). Both $\beta$ 'Asp778 and $\beta$ 'Arg830 are highly conserved residues. There are two direct hydrogen bonds to A36, one from $\beta$ 'Ser747 to N6 and second from $\beta$ 'Thr793 to N1. A37 interacts with the carboxylate group of conserved $\beta$ 'Asp745 via ribose 2'OH. The A38 ribose 2'OH also interacts with $\beta^{\prime} \operatorname{Asp} 745$.

The interactions with D-loop vary across all structures in the initial recognition state, indicating the dynamic nature of these contacts. Here, the $\alpha 1$ ' domain participates in hydrogen bonds to G19 and/or U20 via $\alpha^{\prime} \operatorname{Arg} 45$ and/or $\alpha^{\prime}$ Arg56, $\alpha^{\prime}$ Asp37. Inconsistent pattern of interactions is also present in binding with the T-loop. This region primarily implicates $\alpha 1$ 'Asn64 residues and, in some instances, $\alpha 1$ 'Ala57 and/or $\alpha 1$ 'Arg45 that form hydrogen bonds with C57 and G58.

\section{tRNA ${ }^{\text {Phe }}$ binding in preaminoacylation complex}

The $M t$ FRSt/F-AMS2 structure illustrates the tRNA binding where the entire tRNA molecule is ordered, with the anticodon region firmly bound and the acceptor arm approaching the active site containing the F-AMS - a phenylalanine adenylate analog (Fig. 6). In this state the enzyme continues to interact with the anticodon region via domain $\beta 3$ ', maintaining all interactions described above for the initial recognition state. D-loop G19 and U20 bind to the $\alpha 1$ ' domain with an inconsistent pattern of interactions seen in the four structural representations. The T-loop is engaged as described above and variable loop contributes one stabilizing interaction via the G46 - $\alpha$ 'Thr32 hydrogen bond.

New features are observed as well in the investigated complex. For example, the phosphoryl group of A39 interacts with two cations. One of them links to $\beta$ 'Glu807 and two water molecules, the other binds to $\beta$ 'Phe743 and two water molecules. This latter region bridges the anticodon loop with the D-loop arm, as the magnesium-coordinating water molecules and $\beta$ 'Phe743 main chain amide group bind to phosphoryl group of G27 and $\beta 3$ 'Glu807 main chain interacts with the phosphoryl group of C26. C26 and G25 2'OH groups interact with main chain carbonyl oxygen atoms 
of $\beta$ 'Ala741 and $\beta^{\prime}$ 'Leu805. Additional hydrogen bonds link $\beta$ 'Pro738 to 2'OH of C11 and $\beta$ 'Thr804 to G25, with the latter OG1 - N2 interaction being the only one involving a D-loop base. Then, three hydrogen bonds connect the backbone of D-loop (G10, C11 and U12) to the $\beta 2$ s1 subdomain ( $\beta$ Ser578 and $\beta$ Arg579). All the contacts with the D-loop arm (G10, C11, U12, G25, C26) exist only in the productive binding of the tRNA molecule.

The most significant structural change, besides tighter contacts with the D-loop arm, is the stabilization of the acceptor arm and 3' end. This region becomes ordered and the CCA3' motif is inserted into acylation site. This is possible because the $\alpha 2$ domain rearranges (Fig. 6). Specifically, the $3_{10}$-helix $\eta 2$ and its short flanking sections (residues 151-159) unfold into a loop-like structure. Residues $\alpha$ Gln158, $\alpha$ Glu157 and $\alpha$ Asp159 that in other structures face the CCA binding pocket dramatically change their positions providing access to the otherwise blocked active site. Thus, this section of the protein serves as a 3' end gate. Another residue that moves significantly is $\alpha$ His152, which relocates closer to the binding pocket. There are two consequences of these shifts - the first movement, particularly that of $\alpha$ Asp 159 , creates a new magnesium binding site, while the second positions $\alpha$ His 152 for the interaction with the RNA backbone.

The very well-ordered magnesium ion is hexagonally coordinated by five water molecules and the carboxyl group of $\alpha$ Asp203. The water molecules bridge the protein and the tRNA 3' end, as they interact with $\alpha \mathrm{Thr} 202, \alpha \mathrm{Gln} 158$ (main chain), $\alpha \mathrm{Asp} 159$ (main chain and side chain) as well as all three CCA bases. This rich network of water-mediated contacts appears to be crucial for tRNA anchoring in the active site as there are only two direct hydrogen bonds contributing to the binding: A77 phosphoryl group interacts with $\alpha$ His 152 and its purine N6 atom binds to the $\alpha$ Asp159 main chain. The magnesium ion might be therefore essential for proper positioning of the A76 ribose, which approaches the imino moiety of the ligand within the 3.0 A distance of nitrogen atom to 2'OH. The observed geometry between the acyl donor (F-AMS) and acceptor (tRNA), observed for the first time for any aaRS, mimics the reaction stage where the 2 ' $\mathrm{OH}$ attacks the carbonyl carbon atom and is fully consistent with the accepted mechanism for FRS ${ }^{30,31}$.

\section{MtFRS vs other bacterial homologs}

FRS homologs from E. coli (EcFRS), S. haemoliticus (ShFRS), P. aeruginosa (PaFRS), and the most extensively studied from $T$. thermophilus (TtFRS) have been characterized structurally as 
functional heterotetramers (Table 1). Overall organization of these proteins is identical to that of MtFRS, however superpositions of the entire chains lead to high RMSDs due to the movements of the individual subdomains.

Several features of the adenylation active site are common between the four proteins. In particular, the residues interacting with the L-Phe or adenylate ligands are mostly conserved and superpose well, especially in the vicinity of the amino acid moiety. The exceptions include $\alpha$ His 175 substitution by $\alpha \mathrm{Gln} 169$ in EcFRS associated with a different confirmation of the L-Phe ligand and inhibitor-induced adjustments to the equivalents of Gln180 in ShFRS and PaFRS. The region binding the nucleotide is less aligned. Interestingly, the reorganization of the $\alpha 2$ domain upon adenylate binding vs Phe binding varies between the homologs. While in EcFRS, PaFRS, and ShFRS the conformation of ligand binding fragment of the $\alpha 2$ domain appears to follow the $M t$ FRS pattern, being a more compact in the presence of a nucleotide (E.coli) and an open without it (P.aeruginosa, S. haemoliticus), such a change is less pronounced in TtFRS. A small shift is only observed in the $\beta 4-\beta 5$ loop, the $\beta 8 \downarrow \beta 7 \uparrow \beta 6 \downarrow$ subsection of the central $\beta$-sheet remains intact (Fig. 6). The structure is not much different when $T t$ FRS is in complex with adenylate and tRNA.

The editing domain, encompassed within the $\beta 1$ s 4 subdomain, also bears high level of similarity (Fig. 6). The residues lining the amino acid binding pocket, $M t \beta H i s 275$, Mt $\beta$ Leu300, Mt $\beta$ Gly325, Mt $\beta$ Glu344 and Mt $\beta$ Pro273 are conserved in TtFRS, EcFRS, PaFRS, and ShFRS. Mt $\beta$ Gly324, Mt $\beta$ Met327, Mt $\beta$ Ala366 and MtTyr370 are sometimes replaced by similar residues (Supplemental Fig. S1). The most striking discrepancy is the presence of $M t \beta \operatorname{Trp} 348$. This residue is unique to $M$. tuberculosis homolog, all other sequences contain Phe. The indole nitrogen atom is perfectly positioned to form a hydrogen bond with the ligand, possibly switching the amino acid recognition from repulsive to attractive interactions and providing distinct opportunities for selective inhibitors targeting the editing domain.

T. thermophilus is the only homolog allowing for comparisons of the tRNA binding. As in MtFRS, the anticodon recognition is achieved by binding to the $\beta 3$ ' domain, but the interactions with tRNA vary between the two proteins due to sequence variations. Mt $\beta$ 'Ser747 and Mt $\beta$ 'Thr793 and are both replaced by alanine residues (Tt $\beta$ 'Ala698 and Tt $\beta$ 'Ala744, respectively), leading two the loss of direct hydrogen bonds to A36 equivalent (TtA35) and a potential (water molecules are not modeled in the $T t$ FRSt structures) water-mediated contact with G35 (TtG34). Alanine residues in these positions are 
more common in homologs than Thr and Ser (Supplemental Fig. S1). Additional hydrogen bond bridging to the RNA backbone is eliminated by another non-conserved substitution Mt $\beta$ 'Gln784/Tt $\beta$ 'Pro735. Residues Mt $\beta$ 'Asp745, Mt $\beta$ 'Phe777, Mt $\beta$ 'Asp778 and Mt $\beta$ 'Arg830 are conserved (Tt $\beta^{\prime}$ Asp696, Tt $\beta$ 'Phe728, Tt $\beta$ 'Asp729 and Tt ' $^{\prime}$ Arg780) and bind the tRNA molecule, albeit in somewhat different fashion, and Tt $\beta$ 'Tyr731 stacks against G34 as $M t \beta$ 'Phe780 does against G35.

In the two TtFRS models with tRNA, the 3' end appears to be inserted into the active site of the acylation domain. However, neither of the modeled states illustrates the reaction-ready position of the tRNA molecule observed in MtFRSt/FAMS2. In the TtFRSt complex, the 3' end adenine moiety occupies the adenylate binding pocket, it is of note, however, that the electron density map for the acceptor arm is poor and therefore the accuracy of the model raises some concerns. In the $T t$ FRSt/adenylate structure the 3' end nucleotide faces the solvent. In neither of the structures, the region equivalent to the 3' end gate is significantly reorganized to facilitate tRNA binding. It thus suggests that in the observed states, the TtFRS 3 ' end gate remains closed, preventing the proper positioning of CCA. Of note, while the $\eta 2$ sequence is conserved in FRSs, the following three amino acids are not: MtFRS contains the GEQ segment (residues 156-158) while TtFRS has DMW. It is not only folded differently, but also is positioned closer to the ligand binding pocket, with $\alpha \operatorname{Trp} 149$ facing the lumen and overlapping with the 3' end adenine of tRNA. Another feature distinct for TtFRS is the replacement of MtaAsp203 with $T t$ Glu206, indicating that the potential magnesium binding site created during the aminoacylation reaction may look somewhat different. In other bacterial homologs the sequence of 3' end gate region varies and adopts either the TtFRS state (EcFRS) or $M t$ FRSt/FAMS2 state (StFRS, PaFRS). While they preserve Asp corresponding to MtaAsp203, neither binds a metal cation in the magnesium site. Given the lack of any obvious common patterns guiding the conformation of the 3' end gate, such as the tRNA presence, magnesium coordination, ligand binding or sequence motifs, it appears that its state might be governed by cellular conditions these enzymes must function in.

\section{MtFRS vs human FRSs}

Humans have two FRSs, cytoplasmic and mitochondrial, both of which have had their structures determined (Table 1). The former enzyme (hcFRS) resembles MtFRS $(\alpha \beta)_{2}$ heterotetramer but it 
lacks the anticodon binding domain and contains an extra domain in the $\alpha$ subunit. The mitochondrial variant (hmFRS), characterized in several states, is a monomeric chimera of the bacterial $\alpha 2$ catalytic module and anticodon binding domain $\beta 3$. HmFRS exists in two states: closed in the absence of tRNA, where the $\beta 3$ domain blocks the access to $\alpha 2$, and open, which mimics the domains arrangement seen in bacterial $(\alpha \beta)_{2}$ complexes ${ }^{10}$. While overall organization and sequences of the human enzymes are distinct from $M t \mathrm{FRS}$, the corresponding domains superpose well. The aminoacylation domain of hmFRS adopts conformation similar to that of $M t$ FRSt/FAMS2, with the $\beta 7-\beta 8$ hairpin equivalent closed over the pocket and the 3' end gate open regardless of the ligand/tRNA binding status. The cytosolic homolog, characterized in the presence of L-Phe, remains more open, as seen in the MtFRSt/F complexes, with its 3' end gate open as well.

The aminoacylation sites of the human enzymes, especially hmFRS, share high similarity with MtFRS (Fig. 7). Several residues lining the adenylate binding site are conserved. In hmFRS the only exception include replacement of MtaVal286, sitting deep in the L-Phe pocket, by hmMet258. HcMtFRS is more variable, with several substitutions in the L-Phe binding site: Mt $\alpha$ Phe257/hc $\alpha$ Tyr412, Mt $\alpha$ Phe255/hc $\alpha$ Asn410, Mt $\alpha$ Val286/hc $\alpha$ Phe438, Mt $\alpha$ Gly282/hc $\alpha$ Asn434, and MtaGln180/hcaSer332. These residues are located in the L-Phe binding cavity, which is generally better aligned between the homologs than the adenine-dedicated section.

The editing domain of hcFRS is differently organized in terms of the secondary structure composition, and consequently some of the residues lining the cavity come from different structural elements than in $M t \mathrm{FRS}$, making the architecture of this site substantially different. In particular, the side chain of Mt $\beta$ Tyr370 is in three-dimensional context replaced by hc $\beta A r g 118$ and the role of $M t \beta \operatorname{Trp} 348$ is played by hc $\beta$ Thr256 and the surface created by Mt $\beta$ Ala366 is lined with hc $\beta$ Arg 154 . Also, some residues contributed by analogous secondary structures differ: $M t \beta H i s 275$ is replaced by hc $\beta$ Gly160 while MtßGly328 is mutated to hc $\beta$ Asn 238 .

The tRNA anticodon interactions with hmFRS mostly resemble those found in MtFRS including recognition of the wobble base and the key residues are conserved or replaced by similar side chains (Mt $\beta$ 'Thr793 to hmCys377 or Mt $\beta$ 'Phe777 to hmIle363). The hydrogen bond linking the protein with the RNA backbone via $M t \beta$ 'Gln784 (replaced by hmLys370) is eliminated due the conformational rearrangement of this protein segment. 


\section{Functional implications}

All tRNAs must have similar shape to fit into ribosomal translation machinery and have identical CCA3' end to deliver amino acid to the ribosome active site. The tRNAs seem to have common origin and have significant sequence conservation, as they were generated from repeating sequences and inverted repeats ${ }^{32}$. During evolution these tRNAs differentiated into over 20 amino acid specific groups. It is believed that aaRSs emerged together with tRNAs and they coevolved, making aaRSs one of the oldest enzymes to arise. Today's aaRS utilize different scaffolds, tRNA recognition strategies and catalytic mechanisms to catalyze aminoacylation reaction. In bacteria, each enzyme must specifically recognize tRNA(s) specific for a single amino acid from a pool of some 30 plus tRNAs.

So how aaRSs enzymes make the connection? The amino acid specific isoacceptor tRNAs may have up to six different codons and these tRNAs are aminoacylated by a single enzyme. At the same time, amino acid must be delivered to adenosine ribose at 3' end, about $65 \AA$ away from the anticodon. Therefore, for aaRSs codon recognition may not be the easiest solution to accomplish this.

It was proposed that a code exist equivalent to the "genetic code" in the interactions between aaRS and tRNAs. This code was linked to amino acid properties. One hypothesis proposed that aaRS emerged first as ancestor urzyme-like synthetase that initially recognized only the acceptor stem and later evolved to include other parts of tRNA to increase specificity. Urzymes correspond to conserved cores of both classes of aaRSs lacking their anticodon-binding domains. Surprisingly, urzymes catalyze amino acid activation and acyl transfer with affinities similar to full length aaRS ${ }^{33}$. The argument for first aaRS to recognize tRNA acceptor stems rather than anticodons, is in agreement with the idea that originally proteins were coded by an "operational RNA code" located in the acceptor stem ${ }^{34,35}$.

Structural studies of aaRS complexes with tRNA showed, that there are two groups of enzymes, one that recognizes anticodon loop and stem to discriminate between cognate and non-cognate tRNA and the other that does not. Earlier studies of the yeast FRS binding to tRNA ${ }^{\text {Phe }}$ identified five bases that were important for selectivity in positions $20,35,36,37$ and $73{ }^{36}$, implying that FRS belongs to the former category. Two codons code for L-Phe, 3'CUU and 3'UUU, however typically only one isoacceptor tRNA ${ }^{\text {Phe }}$ with 5'GAA anticodon is required to decode both of these two codons, as the wobble $\mathrm{G}$ base can decode both $\mathrm{C}$ and $\mathrm{U}$. This is the case for M. tuberculosis, as it has only one tRNA ${ }^{\text {Phe }}$. Our structures showed direct FRS interactions only with U20, G35, A36 and A37, with U20 
and A37 having very minor contributions. Therefore, the role of A37 and A73 must be indirectly affecting the structure of tRNA that allows it to make the other interactions.

Our data confirm the interaction of FRS with the anticodon loop and stem, yet no anticodon sequence is recognized. The key feature of the initial detection is binding to the G35 wobble base, the least important in codon-anticodon pairing. With the most extensive interactions with $\beta 3$ ' domain residues G35 appears to be the primary discriminator with some additional selectivity provided by A36, A37, G19 and U20. We believe that the recognition of the anticodon loop and stem is governed by the structural motif of stacked six purine bases (unique for tRNA ${ }^{\text {Phe }}$ in $M$. tuberculosis), which positions tRNA and FRS to make additional contacts, including D- and T-loops. This interface is

preserved across the FRS family, including much simplified version of the enzyme found in human mitochondria. FRS does not interact with the acceptor stem at all, in fact in the initial recognition step the acceptor stem is disordered and the access to active site is often blocked by the enzyme itself. Completion of the recognition process allows for conformational changes in the 3' gate permitting binding of the CCA3' region into the active site.

It seems FRS lost its need to read the CCA stem sequence. This is because other parts of tRNA ${ }^{\text {Phe }}$ provide unique signatures sufficient to allow FRS to distinguish it from other tRNAs. FRS is a large, multidomain enzyme that provides many opportunities for interactions and specificity. tRNA molecules also offer a variety of surfaces for interactions. Our results suggest that recognition of the CCA stem might have been essential for ancestral aaRSs to discriminate between tRNAs, but later in the evolution of the protein genetic code new specificity determinants were added while previous were lost. This indirect recognition is one of the most important highlights of biological systems.

\section{Therapeutics development}

With a multidomain structure rich in pockets, equally complex reaction and essentiality FRS represents a golden mine for drug discovery. Structural differences between the active sites of bacterial and human FRS could be exploited to design narrow spectrum inhibitors that would have low toxicity for humans, other animal hosts and their microbiomes. Admittedly, close similarity of the adenylate binding cavity between MtFRS and mitochondrial homolog present a formidable challenge for medicinal chemistry. Therefore, targeting the editing domain, substantially distinct from the cytoplasmic variant and absent in the mitochondrial enzyme may offer an untapped opportunity. 


\section{Methods}

\section{Cloning and expression of M. tuberculosis FRS subunits, PheS and PheT}

The pheS and pheT genes were amplified from genomic DNA of M. tuberculosis H37Rv using the following pairs of primers, respectively: ${ }^{\prime}$ TACTTCCAATCCAATGCCATGTTGTCGCCGGAGGCATTGA, TTATCCACTTCCAATGTTAGGCACCCACCCCGAACGG and 5'TACTTCCAATCCAATGCCATGCGGCTACCCTACAGCTGG, TTATCCACTTCCAATGTTAGCCACGCAGCACGGCACC. PCR product was purified and treated T4 polymerase in presence of dCTP according to Y. Kim et al. ${ }^{37}$. PheS gene was cloned to pMCSG53, while pheT was cloned to pMCSG120. Both vectors contain identical cloning site, pMCSG53 carries ampicillin resistance and ColE1 origin of replication, pMCSG120 retains resistance to kanamycin and has RSF origin of replication. In the final construct the PheS sequence is amended on $\mathrm{N}$-terminus with $\mathrm{His}_{6}$ affinity tag and Tobacco Etch Virus (TEV) protease cleavage site.

Plasmids PheS-pMCSG53 and PheT-pMCSG120 were co-transformed to E. coli BL21-Gold (DE3) and grown overnight in LB medium supplemented with $40 \mathrm{mM} \mathrm{K}{ }_{2} \mathrm{HPO}_{4}$ at $37^{\circ} \mathrm{C} / 220 \mathrm{rpm}$ with selection against ampicillin $(150 \mu \mathrm{g} / \mathrm{mL})$ and kanamycin $(100 \mu \mathrm{g} / \mathrm{mL})$. The following morning the culture was diluted 1:100 into LB media with the same concentration of antibiotics and grown at $37^{\circ} \mathrm{C} / 190 \mathrm{rpm}$ till OD600 reached about 1.0 followed by cooling of media to about $20^{\circ} \mathrm{C}$. At this point the media was supplemented with: IPTG $0.25 \mathrm{mM}, \mathrm{K}_{2} \mathrm{HPO}_{4} 40 \mathrm{mM}$, glucose $0.5 \%$. The culture was grown for another 20 hours at $18^{\circ} \mathrm{C} / 190 \mathrm{rpm}$ followed by cells harvest and resuspension in buffer A (50 mM HEPES/NaOH, pH 8.0, 0.5 M NaCl, $20 \mathrm{mM}$ imidazole, $\mathrm{pH} 8.0,5 \%$ glycerol, $1 \mathrm{mM}$ TCEP). The cell suspension was frozen and stored at $-80^{\circ} \mathrm{C}$.

\section{Synthesis of M. tuberculosis $t R N A^{\text {Phe }}(G A A)$ precursor}

The isoacceptor tRNA ${ }^{\text {Phe }}(\mathrm{GAA})$ recognizing specifically UUC codon and its truncated derivatives lacking the 3'-terminal adenosines were prepared as described previously by Sherlin et al. ${ }^{38}$. Oligonucleotides used for construction of the tRNA $^{\text {Phe }}$ gene are: 5'-AATTCCTGC 
AGTAATACGACTCACTATAGGCCAGGTAGCTCAGTCGGTATGAGC-3'， 5'-mUmGGTGGCCAGGGGCGGGATCGAACCGCCGACCTTCCGCTTTTCAGGCGGACGCTCATA-

CCGAC-3' and 5'-mGmGTGGCCAGGGGCGGGATCGAACCGCCGACCTTCCGCTTTTCAGGCGGACGCTCATACCGAC-3', where $\mathrm{mU}$ and $\mathrm{mG}$ represent the 2'-O-methyl nucleotides and the underlined portions represent the overlapped segment. Bold type indicates the T7 RNA polymerase promoter. To synthesize these transcripts, $10 \mu \mathrm{g} / \mathrm{mL}$ duplex DNA template in a solution containing $200 \mathrm{mM}$ HEPES/KOH (pH 7.5), $30 \mathrm{mM} \mathrm{MgCl}$, 2 mM spermidine, $40 \mathrm{mM}$ DTT, $6 \mathrm{mM}$ each ATP, CTP, GTP and UTP was incubated with $100 \mu \mathrm{g} / \mathrm{mL}$ T7 RNA polymerase, $5 \mathrm{U} / \mathrm{mL}$ inorganic pyrophosphatase, $50 \mathrm{U} / \mathrm{mL}$ SUPERase In RNase Inhibitor (Invitrogen) in a $8 \mathrm{~mL}$ reaction for $5 \mathrm{~h}$ at $37^{\circ} \mathrm{C}$. DNA template was removed by addition of $50 \mathrm{U} / \mathrm{mL}$ RQ1 RNase-free DNase (Promega) and incubation for $1 \mathrm{~h}$ at $37^{\circ} \mathrm{C}$, followed by phenol-chloroform extraction. The tRNA was recovered from the aqueous phase by ethanol precipitation and then fractionated on DE-52 (Whatman) column as described before ${ }^{38}$. Integrity of the purified tRNA was confirmed by electrophoresis on a 10\% denaturing polyacrylamide gel. The tRNA was dialyzed into $20 \mathrm{mM}$ HEPES/KOH (pH 7.5) $150 \mathrm{mM} \mathrm{KCl}$ and $5 \mathrm{mM} \mathrm{MgCl}_{2}$ buffer prior to further use. The fraction of tRNA capable of being aminoacylated by $M t$ FRS was determined by assays under conditions giving maximal amino acid incorporation (approximately ten-fold molar excess of enzyme over tRNA).

\section{FRS purification and crystallization}

Frozen cells were thawed and sonicated ( 5 min total time, $130 \mathrm{~W}$ power output) and spun at $30,000 \times \mathrm{g}$ at $4^{\circ} \mathrm{C}$ for $1 \mathrm{~h}$. The initial $\mathrm{Ni}^{2+}$ affinity purification step was performed using a $2.5-\mathrm{cm} \times$ 10-cm Flex-Column connected to a Van-Man vacuum manifold (Promega) ${ }^{39}$. Briefly: supernatant was loaded on $3 \mathrm{~mL} \mathrm{Ni}^{2+}$ Sepharose (GE Healthcare Life Sciences) equilibrated with buffer A and mixed thoroughly with the resin. Vacuum of 15 p.s.i. was used to speed removal of supernatant as well as wash out of unbound proteins (150 $\mathrm{mL}$ buffer A supplemented to $50 \mathrm{mM}$ imidazole). The FRS complex was eluted with $15 \mathrm{~mL}$ buffer A supplemented with $500 \mathrm{mM}$ imidazole, pH 8.0. The eluate was concentrated to about $2 \mathrm{~mL}$ and loaded on a Superdex 200 16/70 size exclusion column (GE Healthcare Life Sciences) equilibrated with buffer A. Fractions containing the FRS complex were collected, and buffer A was replaced with crystallization buffer (20 mM HEPES/ NaOH, pH 8.0, $150 \mathrm{mM} \mathrm{KCl}$ ) on Amicon 30-kDa-cutoff concentrators (Millipore) followed by overnight incubation at $4^{\circ} \mathrm{C}$ with synthetic tRNA ${ }^{\text {Phe }}$. The molar ratio of tRNA ${ }^{\text {Phe }}$ to FRS heterotetramer $(\alpha \beta)_{2}$ was $2: 1$. 
Protein-tRNA complex was concentrated to $\sim 20 \mathrm{mg} / \mathrm{mL}$ of complex. The ligands were added at molar ratios $4: 1$. Crystallization experiments were performed at $16^{\circ} \mathrm{C}$ using the sitting-drop vapor-diffusion method in 96-well CrystalQuick plates (Greiner Bio-One, Monroe, NC) with the help of the Mosquito liquid dispenser (TTP LabTech, Cambridge, MA). Crystallizations trials were performed using protein-tRNA to matrix ratio 1:1 (800 $\mathrm{nL}$ drops). Crystallization and cryocooling conditions are given in Table 1.

\section{X-ray data collection, structure determination, refinement and analysis}

Prior to data collection at $100 \mathrm{~K}$, all cryoprotected crystals were flash-cooled in liquid nitrogen. The x-ray diffraction experiments were carried out at the Structural Biology Center 19-ID beamline at the Advanced Photon Source, Argonne National Laboratory. The diffraction images were recorded on the PILATUS3 X 6M detector. The data sets were processed with the HKL3000 suite ${ }^{40}$. Intensities were converted to structure factor amplitudes in the Ctruncate program ${ }^{41,42}$ from the CCP4 package ${ }^{43}$. The data collection and processing statistics are given in Table 1.

The initial FRS structure was solved by molecular replacement using monomeric units of E. coli FRS as a search template (PDB id 3PCO) against the dataset from low resolution apo crystals of MtFRS. The FRSt/F1 structure was determined using the partially built apo model. The search of tRNA molecule from $T$. thermophilus FRS complex structure (PDB id 1EIY) failed so the entire nucleic acid molecule has been built manually from scratch. The subsequent structures were determined using the FRSt/F1 model. The structures were refined by manual corrections in Coot ${ }^{44}$ and crystallographic refinement in Refmac ${ }^{45}$ and Phenix ${ }^{46}$. The refinement statistics are given in Table 1. The structure has been analyzed using Chimera ${ }^{47}$, SNAP (for protein-RNA interactions, snap.x3DNA.org), and the RNA-align server (for RNA superposition) ${ }^{48}$. Some of the atoms that appeared to be cations could not be confidently identified and have been modeled as unknown atoms UNX. The atomic coordinates and structure factors have been deposited in the PDB under accession codes 7KA0, 7KAB, 7K9M, 7K98.

\section{Aminoacylation of precursor of M. tuberculosis $t R N A^{\text {Phe }}$}

Purified M.tuberculosis tRNA transcripts lacking the 3'-terminal adenosine were ${ }^{32} \mathrm{P}$-labeled with yeast ATP/CTP tRNA nucleotidyl transferase in the presence of $\left[\alpha-{ }^{32} \mathrm{P}\right] \mathrm{ATP}$ as described in Wower 
et al. ${ }^{49}$. Aminoacylations of the 3'- ${ }^{32} \mathrm{P}$-labeled M. tuberculosis $\mathrm{tRNA}^{\text {Phe }}$ transcript were carried out in $50 \mu \mathrm{L}$ of $50 \mathrm{mM}$ HEPES/KOH, pH 7.5- $20 \mathrm{mM} \mathrm{KCl-} 10 \mathrm{mM} \mathrm{MgCl}$ - $^{-} 100 \mu \mathrm{g} / \mathrm{mL}$ BSA- 5 mM DTT$4 \mathrm{mM}$ ATP- $0.2 \mathrm{mM}$ L-Phe and $50 \mathrm{nM}$ tRNA transcript. Reactions were initiated by addition of a purified $M$. tuberculosis phenylalanyl-tRNA synthetase to final concentration of $0.7 \mu \mathrm{M}$ and incubated for $30 \mathrm{~min}$ at $37^{\circ} \mathrm{C}$. The extent of aminoacylation was analyzed by gel-shift electrophoresis according to Varshney et al. ${ }^{50}$. Prior to electrophoresis on a $10 \%$ acid/7 M urea polyacrylamide gel, 10 pmoles of uncharged and aminoacylated tRNA (Phe-tRNA) were completely digested with 2 U of RNase T1 (Worthington) in $10 \mathrm{mM}$ sodium acetate ( $\mathrm{pH} 4.5)$. The amount of radioactivity in the bands corresponding to uncharged and aminoacylated CCACCA oligonucleotides was quantitated by phosphorimaging and/or liquid scintillation counting of the excised strips of the polyacrylamide gel.

The value of $\mathrm{IC}_{50}$ was determined at $1.5 \mu \mathrm{M}$ concentration of phenylalanine over a range of inhibitor concentration from zero to $200 \mathrm{nM}$. According to the Cheng-Prusoff equation, $\mathrm{IC}_{50}$ values approximate $K_{i}$ when substrate concentration used in the assay is lower than $K_{m}{ }^{51}$.

\section{Aminoacylation of $E$. coli and yeast $t R N A^{\text {Phe }}$}

Aminoacylation reactions were performed as described above with the following modifications: 3'- ${ }^{32} \mathrm{P}-$ labeled $M$. tuberculosis tRNA ${ }^{\text {Phe }}$ transcripts were replaced either by non-radioactive $M$. tuberculosis $\mathrm{tRNA}^{\text {Phe }}$ transcripts or fully modified E. coli and yeast tRNA ${ }^{\text {Phe }}$ preparations from Sigma. $\left[\alpha{ }^{32} \mathrm{P}\right]$ ATP from PerkinElmer was used instead of non-radioactive ATP. All reactions were quenched in $400 \mathrm{mM}$ sodium acetate $(\mathrm{pH}$ 5.2) with $0.1 \%$ SDS. Aliquots were spotted on prewashed polyethyleneimine-cellulose TLC plates (Macherey and Nagel) and developed in $100 \mathrm{mM}$ ammonium acetate, $5 \%(\mathrm{v} / \mathrm{v})$ acetic acid.

\section{MtFRS inhibition by $F-A M S$}

Aminoacylation assays were carried out by a very sensitive assay that has been described by Bullock et al. ${ }^{52}$. The assay was performed by $10 \mathrm{~min}$ incubation of L-Phe with purified enzyme in $50 \mathrm{mM}$ HEPES/KOH (pH 7.5)- 20 mM KCl- 10 mM MgCl $2-4 \mathrm{mM} \mathrm{ATP/20} \mu \mathrm{M}$ [ $\alpha$ - $\left.{ }^{32} \mathrm{P}\right] \mathrm{ATP}-0.1 \%$

dimethyl sulfoxide in a final volume of $20 \mu \mathrm{L}$ at $37^{\circ} \mathrm{C}$. $\mathrm{K}_{\mathrm{m}}$ for $\mathrm{L}-\mathrm{Phe}$ and $\mathrm{K}_{\mathrm{i}}$ were determined by varying concentrations of amino acid and inhibitor from $0.5-25 \mathrm{mM}$ and from $15-125 \mathrm{nM}$, respectively. The concentration of FRS was $6 \mathrm{nM}$. The reactions were stopped by adding $2.0 \mu \mathrm{L}$ of the reaction mixtures to $3.0 \mu \mathrm{L}$ of a solution containing $400 \mathrm{mM}$ sodium acetate $(\mathrm{pH} 5.0)$ and $0.1 \%$ 
SDS. 2.0 $\mu \mathrm{L}$ aliquots of this mixture were spotted on dry polyethyleneimine (PEI) cellulose plates that have been prewashed in water. Phe-[ $\left.{ }^{32} \mathrm{P}\right] \mathrm{AMP}$ were separated from $\left[\alpha{ }^{-32} \mathrm{P}\right] \mathrm{ATP}$ using $0.1 \mathrm{M}$ ammonium acetate $/ 5 \%$ acetic acid as developing solution, visualized by autoradiograph and quantified by phosphorimaging analysis.

\section{Synthesis of phenylalanine adenylate analog}

$5^{\prime}-O$-[ $N$-(phenylalanine)sulfamoyl] adenosine, Phe-AMS, has been prepared in six steps as previously described ${ }^{53}$.

\section{Conflict of Interest Statement}

All authors declare no competing interest

\section{Acknowledgements}

We truthfully thank the members of the SBC at Argonne National Laboratory for their help with setting beamline and data collection at beamline 19-ID. Funding for this project was provided in part by federal funds from the National Institute of Allergy and Infectious Diseases, National Institutes of Health, Department of Health and Human Services, under Contracts No. HHSN272201200026C and HHSN272201700060C. The use of SBC beamlines at the Advanced Photon Source is supported by the U.S. Department of Energy (DOE) Office of Science and operated for the DOE Office of Science by Argonne National Laboratory under Contract No. DE-AC02-06CH11357. JW was supported by the Hatch program of the National Institute of Food and Agriculture, U.S. Department of Agriculture. We acknowledge support from the Structure-guided Drug Discovery Coalition funded by the Bill \& Melinda Gates Foundation. 
Table 1. FRS structures in the Protein Data Bank.

\begin{tabular}{|c|c|c|c|c|c|}
\hline PDB ID & Source & Structure & $\operatorname{Res}(\AA)$ & $\begin{array}{c}\text { \% Sequence identity } \\
\text { to } M t(\alpha / \beta)^{a}\end{array}$ & reference \\
\hline $1 \mathrm{~B} 70$ & T. thermophilus & $(\alpha / \beta)_{2}$ complex with L-Phe in $\alpha$ & 2.70 & $39 / 34$ & Reshetnikova et al. ${ }^{17}$ \\
\hline $1 \mathrm{~B} 7 \mathrm{Y}$ & T. thermophilus & $\begin{array}{l}(\alpha / \beta)_{2} \text { complex with adenosine- } 5^{\prime}- \\
\text { [phenylalaninol-phosphate] in } \alpha\end{array}$ & 2.50 & & Reshetnikova et al. ${ }^{17}$ \\
\hline $1 \mathrm{EIY}$ & T. thermophilus & $(\alpha / \beta)_{2}$ complex with tRNA ${ }^{\text {Phe }}$ & 3.30 & & Goldur et al. ${ }^{54}$ \\
\hline $1 \mathrm{JJC}$ & T. thermophilus & $\begin{array}{l}(\alpha / \beta)_{2} \text { complex with adenosine- } 5^{\prime}- \\
\text { [phenylalaninyl-phosphate] in } \alpha\end{array}$ & 2.60 & & Fishman et al. ${ }^{55}$ \\
\hline 1PYS & T. thermophilus & $(\alpha / \beta)_{2}$ complex & 2.90 & & Mosyak et al. ${ }^{12}$ \\
\hline $2 \mathrm{AKW}$ & T. thermophilus & $(\alpha / \beta)_{2}$ complex with p-Cl-Phe in $\alpha$ & 2.80 & & Kotik-Kogan et al. ${ }^{18}$ \\
\hline $2 \mathrm{ALY}$ & T. thermophilus & $\begin{array}{l}(\alpha / \beta)_{2} \text { complex with } 5 \text { '- } O-[N-\mathrm{L}- \\
\text { tyrosyl)sulfamoyl]adenosine in } \alpha\end{array}$ & 2.60 & & Kotik-Kogan et al. ${ }^{18}$ \\
\hline 2AMC & T. thermophilus & $(\alpha / \beta)_{2}$ complex with L-Tyr in $\beta$ & 2.70 & & Kotik-Kogan et al. ${ }^{18}$ \\
\hline $2 \mathrm{IY} 5$ & T. thermophilus & $\begin{array}{l}(\alpha / \beta)_{2} \text { complex with tRNA }{ }^{\text {Phe }} \text { and adenosine- } \\
5 \text { '-[phenylalaninol-phosphate] in } \alpha\end{array}$ & 3.10 & & Moor et al. ${ }^{56}$ \\
\hline $3 \mathrm{HFZ}$ & T. thermophilus & $(\alpha / \beta)_{2}$ complex with m-L-Tyr in $\alpha$ & 2.90 & & Klipcan et al. ${ }^{57}$ \\
\hline $3 \mathrm{TEH}$ & T. thermophilus & $\begin{array}{c}(\alpha / \beta)_{2} \text { complex with } 3,4- \\
\text { dihydroxyphenylalanine in } \alpha\end{array}$ & 2.85 & & Moor et al. ${ }^{58}$ \\
\hline 4TVA & T. thermophilus & $\begin{array}{l}(\alpha / \beta)_{2} \text { complex with puromycin in } \beta \text { and L- } \\
\text { Phe in } \alpha\end{array}$ & 2.60 & & Tworowski et al. ${ }^{59}$ \\
\hline 2RHQ & S. haemolyticus & $\begin{array}{l}(\alpha / \beta)_{2} \text { complex with 1-\{3-[(4-pyridin-2- } \\
\text { ylpiperazin-1-yl)sulfonyl]phenyl }\}-3-(1,3- \\
\text { thiazol-2-yl)urea in } \alpha, 6 \text { mutations in } \beta\end{array}$ & 2.20 & $41 / 31$ & Evdokimov et al. ${ }^{14}$ \\
\hline 2RHS & S. haemolyticus & $\begin{array}{l}(\alpha / \beta)_{2} \text { complex with } 1-\{3-[(4-\text { pyridin-2- } \\
\text { ylpiperazin-1-yl)sulfonyl]phenyl }\}-3-(1,3- \\
\text { thiazol-2-yl }) \text { urea in } \alpha, 5 \text { mutations in } \beta\end{array}$ & 2.20 & & Evdokimov et al. ${ }^{14}$ \\
\hline $3 \mathrm{PCO}$ & E.coli & $(\alpha / \beta)_{2}$ complex with L-Phe and AMP in $\alpha$ & 3.02 & $45 / 31$ & Mermershtain et al. ${ }^{16}$ \\
\hline $4 \mathrm{P} 71$ & P.aeruginosa & $(\alpha / \beta)_{2}$ complex & 2.79 & $43 / 32$ & Abibi et al. ${ }^{60}$ \\
\hline $4 \mathrm{P} 72$ & P. aeruginosa & $\begin{array}{l}(\alpha / \beta)_{2} \text { complex with compound 2-\{3-[(4- } \\
\text { chloropyridin-2-yl)amino]phenoxy }\}-\mathrm{N}- \\
\text { methylacetamide in } \alpha\end{array}$ & 2.62 & & Abibi et al. ${ }^{60}$ \\
\hline $4 \mathrm{P} 73$ & P.aeruginosa & $\begin{array}{c}(\alpha / \beta)_{2} \text { complex with compound } 1-\{3-[(4- \\
\text { pyridin-2-ylpiperazin-1-yl)sulfonyl]phenyl }\}- \\
\text { 3-(1,3-thiazol-2-yl)urea in } \alpha \\
\end{array}$ & 3.03 & & Abibi et al. ${ }^{60}$ \\
\hline $4 \mathrm{P} 74$ & P. aeruginosa & $\begin{array}{c}(\alpha / \beta)_{2} \text { complex with compound N-[(3S)-1,1- } \\
\text { dioxidotetrahydrothiophen-3-yl]-2-[(4- } \\
\text { methylphenoxy)methyl]-1,3-thiazole-4- } \\
\text { carboxamide in } \alpha\end{array}$ & 2.70 & & Abibi et al. ${ }^{60}$ \\
\hline $4 \mathrm{P} 75$ & P. aeruginosa & $\begin{array}{l}(\alpha / \beta)_{2} \text { complex with compound 3-(3- } \\
\text { methoxyphenyl)-5-(trifluoromethyl)-1H- } \\
\text { pyrazole in } \alpha\end{array}$ & 2.96 & & Abibi et al. ${ }^{60}$ \\
\hline 3ICA & P. gingivalis & $\beta$ subunit fragment & 2.44 & $36 / 27$ & Fan et al., unpublished \\
\hline $3 \mathrm{IG} 2$ & B. fragilis & $\beta$ subunit fragment & 2.09 & $38 / 28$ & $\begin{array}{c}\text { Stein } \text { et al., } \\
\text { unpublished }\end{array}$ \\
\hline $2 \mathrm{CXI}$ & P. horikoshii & $\beta$ subunit fragment, 9 mutations & 1.94 & $19 / 13$ & Sasaki et al. ${ }^{13}$ \\
\hline 3CMQ & H. sapiens & $\begin{array}{l}\text { Mitochondrial monomer with adenosine-5'- } \\
\text { [phenylalaninyl-phosphate] }\end{array}$ & 2.20 & $27,28^{b}$ & Klipcan et al. ${ }^{61}$ \\
\hline $3 \mathrm{HFV}$ & H. sapiens & Mitochondrial monomer with m-L-Tyr & 2.60 & & Klipcan et al. ${ }^{57}$ \\
\hline
\end{tabular}




\begin{tabular}{|c|c|c|c|c|c|}
\hline 3TEG & H. sapiens & $\begin{array}{c}\text { Mitochondrial monomer with 3,4- } \\
\text { dihydroxyphenylalanine }\end{array}$ & 2.20 & & Moor et al. ${ }^{58}$ \\
\hline 3TUP & H. sapiens & Mitochondrial monomer with $T t \mathrm{tRNA}^{\text {Phe }}$ & 3.05 & & Klipcan et al. ${ }^{10}$ \\
\hline $5 \mathrm{MGH}$ & H. sapiens & $\begin{array}{l}\text { Mitochondrial monomer, point mutant with } \\
\text { L-Phe }\end{array}$ & 1.87 & & Kartvelishvili et al. ${ }^{62}$ \\
\hline $5 \mathrm{MGU}$ & H. sapiens & $\begin{array}{l}\text { Mitochondrial monomer, point mutant with } \\
\text { L-Phe }\end{array}$ & 1.89 & & Kartvelishvili et al. ${ }^{62}$ \\
\hline $5 \mathrm{MGW}$ & H. sapiens & $\begin{array}{l}\text { Mitochondrial monomer, point. mutant with } \\
\text { L-Phe }\end{array}$ & 1.46 & & Kartvelishvili et al. ${ }^{62}$ \\
\hline $5 \mathrm{MGV}$ & H. sapiens & Mitochondrial monomer, point mutant & 2.05 & & Kartvelishvili et al. ${ }^{62}$ \\
\hline $3 \mathrm{~L} 4 \mathrm{G}$ & H. sapiens & Cytosolic $(\alpha / \beta)_{2}$ complex with L-Phe in $\alpha$ & 3.30 & $34 / 18$ & Finarov et al. ${ }^{15}$ \\
\hline
\end{tabular}

${ }^{a}$ As determined by Emboss Needle ${ }^{63}$.

${ }^{b}$ The numbers correspond to MtFRS $\alpha$ subunit residues 108 - 341 aligned with human mitochondrial FRS residues 82 -

347 and $M t$ FRS $\beta$ subunit residues 739-831 with 360-451). 
Table 2. Data processing and refinement statistics.

\begin{tabular}{|c|c|c|c|c|}
\hline \multicolumn{5}{|c|}{ Data processing } \\
\hline Structure & FRSt/F1 & FRSt/F2 & FRSt/F-AMS1 & FRSt/F-AMS2 \\
\hline $\begin{array}{l}\text { Crystallization conditions; } \\
\text { cryoprotectant supplement }\end{array}$ & $\begin{array}{c}0.2 \mathrm{M} \mathrm{Li}_{2} \mathrm{SO}_{4}, 0.1 \mathrm{M} \\
\text { HEPES pH 7.5, } 25 \% \\
\text { PEG3350; } \\
10 \% \text { glycerol }\end{array}$ & $\begin{array}{c}14.3 \% \text { PEG20K, } \\
150 \text { mM TAPS } \\
\text { pH 8.5, 5\% 1,2- } \\
\text { propanediol; } \\
20 \% \text { glycerol }\end{array}$ & $\begin{array}{c}0.2 \mathrm{M} \mathrm{Na} / \mathrm{K} \text { tartrate } \\
20 \% \text { PEG3350; } \\
15 \% \text { glycerol }\end{array}$ & $\begin{array}{c}30 \% \mathrm{PEG} 400,0.2 \\
\mathrm{M} \mathrm{MgCl}_{2}, 0.1 \mathrm{M} \\
\text { HEPES pH 7.5; } \\
\text { No cryoprotectant }\end{array}$ \\
\hline Wavelength $(\AA)$ & 0.9793 & 0.9793 & 0.9793 & 0.9793 \\
\hline Resolution range $(\AA)^{a}$ & $\begin{array}{l}2.40-50.00 \\
(2.40-2.44)\end{array}$ & $\begin{array}{l}2.50-30.00 \\
(2.50-2.54)\end{array}$ & $\begin{array}{c}2.50-50.0 \\
(2.50-2.54)\end{array}$ & $\begin{array}{c}2.20-30.0 \\
(2.20-2.24)\end{array}$ \\
\hline Space group & $C 2$ & $C 2$ & $C 2$ & $P 2_{1}$ \\
\hline Unit cell $\left(\AA,^{\circ}\right)$ & $\begin{array}{l}\mathrm{a}=292, \mathrm{~b}=110, \\
\mathrm{c}=128, \beta=100\end{array}$ & $\begin{array}{l}\mathrm{a}=127, \mathrm{~b}=110, \\
\mathrm{c}=148, \beta=105\end{array}$ & $\begin{array}{c}\mathrm{a}=128, \mathrm{~b}=110, \mathrm{c}=147, \\
\beta=103\end{array}$ & $\begin{array}{l}\mathrm{a}=147, \mathrm{~b}=65, \\
\mathrm{c}=193, \beta=109\end{array}$ \\
\hline Unique reflections (merged) & $155,793(7,677)$ & $68,179(3,404)$ & $68,621(3,274)$ & $173,141(8,263)$ \\
\hline Multiplicity & $6.4(5.5)$ & $7.0(5.9)$ & $6.2(3.9)$ & $6.4(6.0)$ \\
\hline Completeness (\%) & $98.9(98.2)$ & $99.3(99.4)$ & $99.0(96.2)$ & $96.9(93.6)$ \\
\hline Mean I/sigma(I) & $14.2(1.78)$ & $17.9(1.10)$ & $13.8(1.60)$ & $21.5(1.56)$ \\
\hline Wilson B-factor $\left(\AA^{2}\right)$ & 32.4 & 52.5 & 31.9 & 45.8 \\
\hline R-merge ${ }^{b}$ & $0.147(1.207)$ & $0.141(1.374)$ & $0.171(0.832)$ & $0.094(1.223)$ \\
\hline $\mathrm{CC} 1 / 2^{c}$ & $0.992(0.673)$ & $(0.487)$ & $0.990(0.522)$ & $(0.596)$ \\
\hline \multicolumn{5}{|c|}{ Refinement } \\
\hline Resolution range $(\AA)$ & $2.40-48.0$ & $2.50-29.69$ & $2.50-47.92$ & $2.19-29.85$ \\
\hline Reflections work/test & $135,987 / 7,094$ & $66,452 / 1,665$ & $63,320 / 3,264$ & $169,565 / 3,498$ \\
\hline $\mathrm{R}_{\text {work }} / \mathrm{R}_{\text {free }}^{d}$ & $0.176 / 0.215$ & $0.187 / 0.213$ & $0.179 / 0.215$ & $0.178 / 0.224$ \\
\hline Number of non-hydrogen atoms & 21,651 & 10,440 & 10,722 & 22,017 \\
\hline macromolecules & 20,563 & 10,237 & 10,292 & 21,241 \\
\hline ligands/solvent & $184 / 904$ & $53 / 150$ & $108 / 322$ & $101 / 675$ \\
\hline Amino acid residues & $339+835+336+835$ & $336+835$ & $339+834$ & $343+834+343+836$ \\
\hline Nucleic acid bases & $66+66$ & 66 & 66 & $77+77$ \\
\hline RMSD(bonds) (̊̊) & 0.003 & 0.002 & 0.002 & 0.007 \\
\hline
\end{tabular}




\begin{tabular}{|l|c|c|c|c|}
\hline RMSD (angles) $\left(^{\circ}\right)$ & 0.599 & 0.478 & 0.504 & 0.915 \\
\hline Ramachandran favored $(\%)$ & 96.65 & 96.39 & 96.83 & 96.85 \\
\hline Ramachandran allowed (\%) & 3.09 & 3.26 & 3.08 & 3.02 \\
\hline Ramachandran outliers (\%) & 0.26 & 0.34 & 0.09 & 0.13 \\
\hline Rotamer outliers (\%) & 2.03 & 1.88 & 0.98 & 2.79 \\
\hline Clashscore & 3.45 & 3.93 & 3.04 & 2.70 \\
\hline Average B-factor $\left(\AA^{2}\right)$ & 65.58 & 99.46 & 56.72 & 61.11 \\
\hline macromolecules & 66.51 & 100.05 & 57.36 & 61.46 \\
\hline ligands & 67.43 & 92.47 & 54.38 & 49.86 \\
\hline solvent & 44.14 & 61.43 & 36.99 & 51.67 \\
\hline Number of TLS groups & 39 & 16 & 11 & 34 \\
\hline PDB ID & $7 \mathrm{KA} 0$ & $7 \mathrm{KAB}$ & $7 \mathrm{~K} 9 \mathrm{M}$ & $7 \mathrm{~K} 98$ \\
\hline
\end{tabular}

${ }^{a}$ Values in parentheses correspond to the highest resolution shell.

${ }^{b}$ Rmerge $=\Sigma \mathrm{h} \Sigma \mathrm{j} \mid \mathrm{Ihj}-<\mathrm{Ih}>\mathrm{I} / \Sigma \mathrm{h} \Sigma \mathrm{j} \mathrm{Ihj}$, where Ihj is the intensity of observation $\mathrm{j}$ of reflection $\mathrm{h}$.

${ }^{c}$ As defined by Karplus and Diederichs ${ }^{64}$.

${ }^{d} \mathrm{R}=\Sigma \mathrm{h}|\mathrm{Fo}|-|\mathrm{Fcl} / \Sigma \mathrm{h}| \mathrm{Fol}$ for all reflections, where $\mathrm{Fo}$ and $\mathrm{Fc}$ are observed and calculated structure factors, respectively. Rfree is calculated analogously for the test reflections, randomly selected and excluded from the refinement.

${ }^{d}$ As defined by Molprobity ${ }^{65}$. 


\section{Figure legends}

Figure 1. MtFRS activity. (A) Aminoacylation of unmodified M. tuberculosis tRNA ${ }^{\text {Phe }}$. The 3'- ${ }^{32} \mathrm{P}-$ labeled tRNA transcript was charged with L-Phe in the presence of purified $M t$ FRS. The extent of aminoacylation was analyzed according to Varshney et al. ${ }^{50}$. Prior to polyacrylamide gel electrophoresis, tRNA and Phe-tRNA were digested with RNase T1 to yield 3'-32P-labeled CCACCA and CCACCA-Phe, respectively. (+) and (-) indicate the presence and the absence of the enzyme. (BD) Synthesis of the Phe-AMP intermediate by $M t$ FRS in the presence of unmodified M.tuberculosis $\operatorname{tRNA}^{\text {Phe }}(\mathrm{B})$, E. coli $\mathrm{tRNA}{ }^{\text {Phe }}(\mathrm{C})$ and yeast $\mathrm{tRNA}^{\text {Phe }}(\mathrm{D})$. Phe- $\left[{ }^{32} \mathrm{P}\right]$ AMP synthesis was monitored by thin layer chromatography. Phe-AMP and ATP denote ${ }^{32} \mathrm{P}$-labeled compounds. F - full reaction; -E, -tRNA and -Phe - control reactions lacking the enzyme, tRNA or amino acid.

Figure 2. Inhibition of MtFRS by F-AMS. (A) Competitive binding of inhibitor with respect to LPhe. Inhibitor concentrations were as follows: $0 \mu \mathrm{M}$ (black line), $15 \mathrm{nM}$ (green line), $50 \mathrm{nM}$ (red line), and $125 \mathrm{nM}$. (B) Phe-[32P]AMP synthesis in the presence of $15 \mathrm{nM}$ inhibitor (corresponding to green line) monitored by thin layer chromatography. The rate of Phe-[ $\left.{ }^{32} \mathrm{P}\right] \mathrm{AMP}$ formation was determined at $37^{\circ} \mathrm{C}$ over $10 \mathrm{~min}$. (C-D) Competitive inhibition of MtFRS by the F-AMS shown in the form of a Lineweaver-Burk plot. $\mathrm{K}_{\mathrm{m}}$ for Phe is $1.78 \mu \mathrm{M}(\mathrm{C})$. The secondary diagram was used to determine the inhibition constant $\mathrm{K}_{\mathrm{i}}$, which is $33 \mathrm{nM}$ (D). (E) Plot of the dose-response curve shows the percentage of initial velocity reduced by various concentrations of the F-AMS. The insert illustrates the inhibition of Phe-[ $\left.{ }^{32} \mathrm{P}\right] \mathrm{AMP}$ synthesis monitored by thin layer chromatography. Dotted lines indicate that the inhibitor has $\mathrm{IC}_{50}$ value of approximately $36 \mathrm{nM}$.

Figure 3. Overall structure of the $M t$ FRS-tRNA ${ }^{\text {Phe }}$ complex. (A) $M t$ FRS-tRNA ${ }^{\text {Phe }}$ heterotetramer with initial tRNA binding $(M t \mathrm{FRS} / \mathrm{F} 1)(\mathrm{B}) M t \mathrm{FRS}^{-t R N A}{ }^{\text {Phe }}$ heterotetramer with productive tRNA binding (MtFRS/F-AMS2). Subunits $\alpha$ shown in purple and coral, subunits $\beta$ shown in grey and teal, tRNA represented as yellow and green ribbon models.

Figure 4. MtFRS domains. (A) Subunit $\alpha$. (B) Subunit $\beta$. (C) Domain boundaries with starting residues numbered above the sequence-representing bars and ending residues shown under the bars. Grey sections depict linkers. 
Figure 5. MtFRS interactions with ligands. (A) L-Phe binding to the aminoacylation site of FRSt/F1. The upper-left insert shows $2 \mathrm{mFo}-\mathrm{DFc}$ electron density map contoured around L-Phe at $1.2 \sigma$. (B) Stereoview of F-AMS binding to the aminoacylation site of FRSt/F-AMS2. (C) Superposition of the aminoacylation site of FRSt/F-AMS2 (purple), FRSt/F-AMS1 (yellow), and FRSt/F1 (coral). (D) F-AMS ligand and its 2mFo-DFc electron density map from the FRSt/F-AMS2 complex contoured at $1.2 \sigma$. Red spheres represent water molecules, a green sphere indicates magnesium ion. Hydrogen bonds are shown are dashed lines.

Figure 6. MtFRS interactions with tRNA. (A) tRNA molecule and interacting residues in $M t$ FRSt/F-AMS2. (B) (B) tRNA molecule and its contacts with the polypeptide chain as seen in MtFRS/F-AMS, (chain C). The interacting residues include those forming hydrogen bonds, participating in base/amino acid pairs and base/amino acid stacks, as defined by the SNAP program (http://forum.x3dna.org/dna-protein-interactions/). The $\mathrm{C} \alpha$ atoms of the interacting residues are shown, colored as in A. Magnesium ions are colored green. Nucleotides have the following colorcoding: G-purple, C-pink, A-green, U-orange. (C) Stereoview of $M t \mathrm{FRS}$ interactions with the anticodon loop. (D) F-AMS (grey), tRNA 3' end (green) and magnesium ion (green sphere) binding in the $M t$ FRSt/F-AMS2 active site shown in 2DFo-Fc electron density map contoured at $1.2 \sigma$. (E) tRNA 3' end interactions in MtFRSt/F-AMS2. (F) Superposition of the tRNA 3' end binding region of $M t \mathrm{FRSt} / \mathrm{F}-\mathrm{AMS} 2$ (purple) and $M t \mathrm{FRSt} / \mathrm{F}-\mathrm{AMS} 1$ (blue).

Figure 7. Comparison of MtFRS with T. thermophilus FRS. (A, B) Comparison of the anticodon binding between $M t$ FRSt/F-AMS2 (green, chain C/E) and TtFRSt/adenylate (yellow, PDB 2IY5). In (A) ribbon representation of the $\beta 3$ ' domain and anticodon loop. In (B) atomic representation of the anticodon loop and interacting residues. For superposition only domain $\beta 3$ ' was used. Labels in black correspond to $M t \mathrm{FRS}$, in yellow to $T t \mathrm{FRS}$. (C) Superposition of the aminoacylation domains from $M t \mathrm{FRSt} / \mathrm{F}$ (coral), MtFRSt/F-AMS1 (blue), TtFRS/F (grey, 1B70), and TtFRS/adenylate (green, 1B7Y). Only select residues are labeled. Parentheses indicate TtFRS. (D) Superposition of the 3' end binding region in $M t \mathrm{FRSt} / \mathrm{F}-\mathrm{AMS} 2$ and $T t \mathrm{FRSt} /$ adenylate. $M t \mathrm{FRS}$ is shown in purple (protein), green (tRNA) and grey (F-AMS). TtFRS is shown in yellow. (E) As in (D) but with the TtFRSt complex (pink). Labels in black correspond to $M t \mathrm{FRS}$, in yellow or pink to TtFRS. (F) Superposition of the 
editing domains ( $\beta 1 \mathrm{~s} 4$ subdomain) from $M t \mathrm{FRSt} / \mathrm{F}-\mathrm{AMS} 2$ (grey) and TtFRS/L-Tyr complex (gold, 2AMC).

Figure 8. Comparison of MtFRS with human FRSs. (A-B) Superposition of the aminoacylation domains from $M t$ FRSt/F-AMS2 (purple) with human mitochondrial (hm) enzyme in complex with adenosine-5'-[phenylalaninyl-phosphate] (3CMQ, olive green) or with $T$. termophilus tRNA ${ }^{\text {Phe }}$, hmFRSt (3TUP, coral). In (B) close-up view of the L-Phe subpocket is shown. (C-D). Superposition of the aminoacylation domains from MtFRSt/F-AMS2 (purple) with human cytosolic enzyme (hcFRS) in complex with L-Phe (3L4G, green). In (D) close-up view of the L-Phe subpocket is shown. (E) Superposition of the anticodon binding region of MtFRSt/F-AMS2 (grey) and hmFRSt (coral). Labels in parentheses correspond to the respective human homologs. (F) Superposition of the editing domains ( $\beta 1 \mathrm{~s} 4$ subdomain) from $M t \mathrm{FRSt} / \mathrm{F}-\mathrm{AMS} 2$ (grey) and hcFRS (3L4G, green). Gold LTyr from the $T t \mathrm{FRS} / \mathrm{L}-\mathrm{Tyr}$ complex marks the amino acid binding site. 
Figure 1.

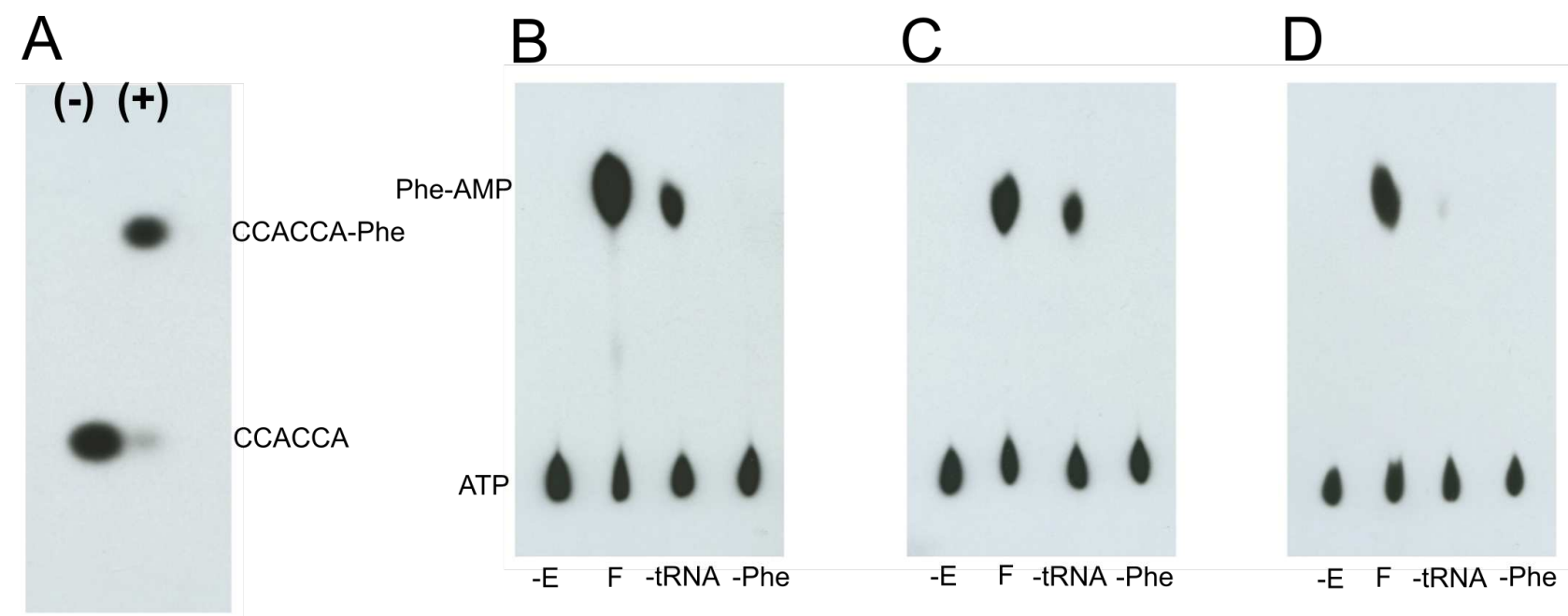


Figure 2.

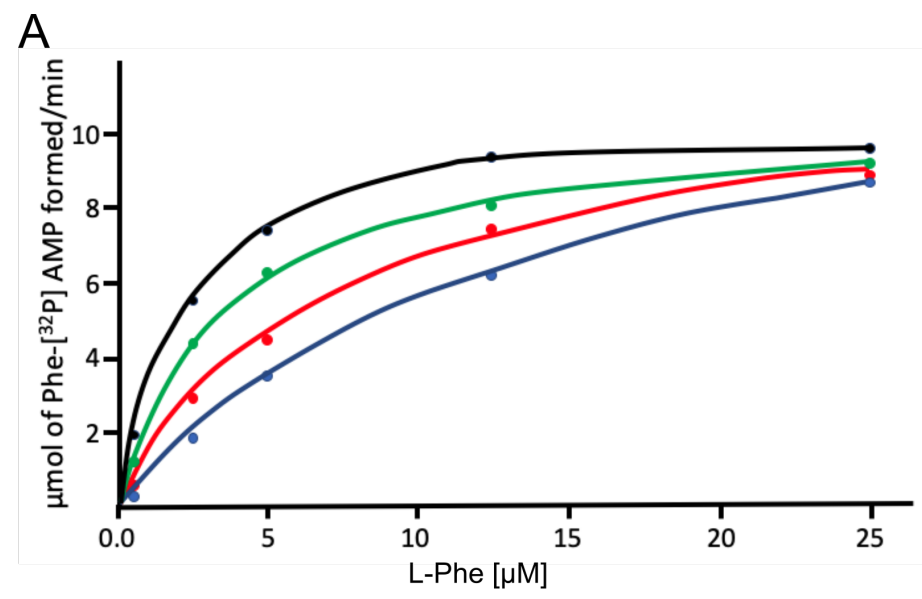

B

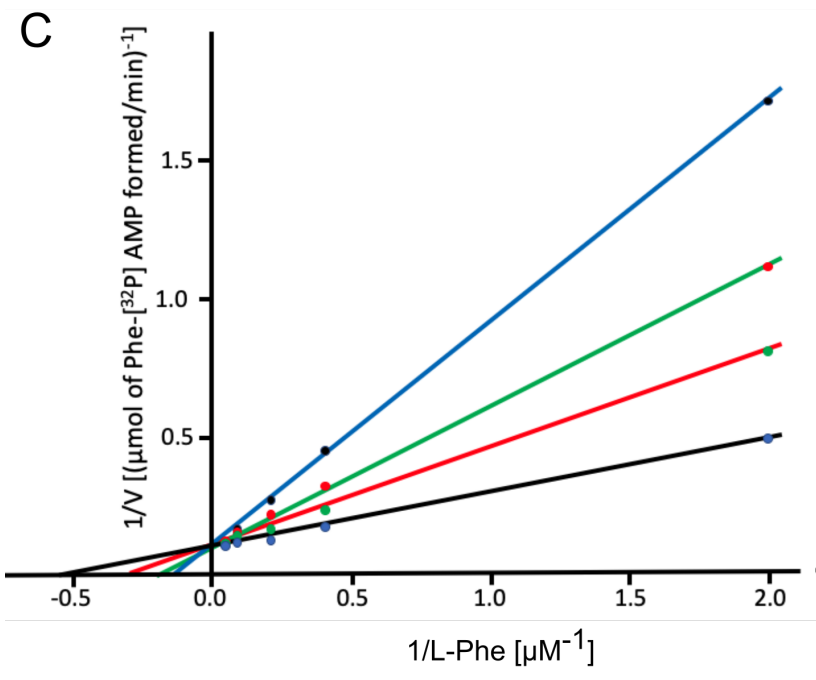

D

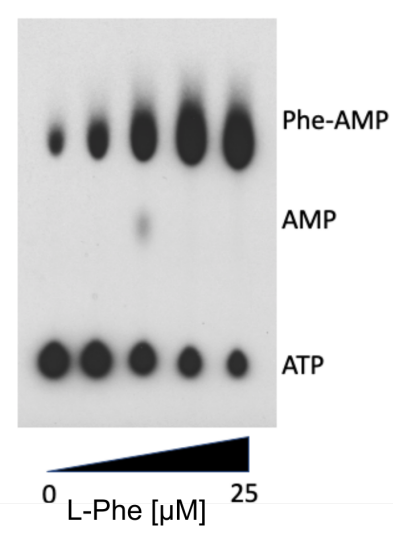

E

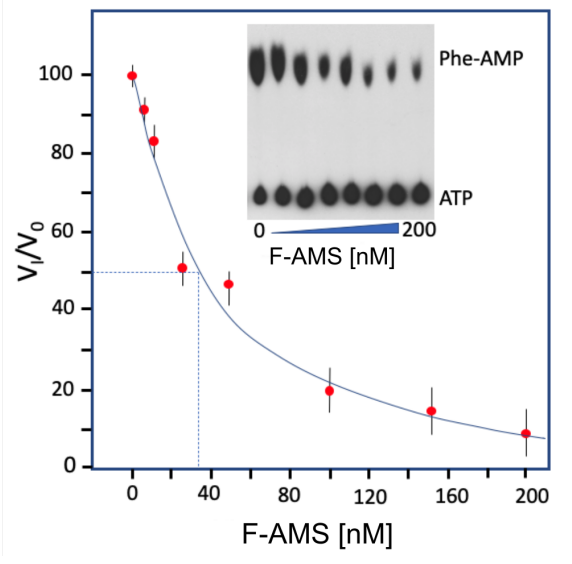


Figure 3.

A

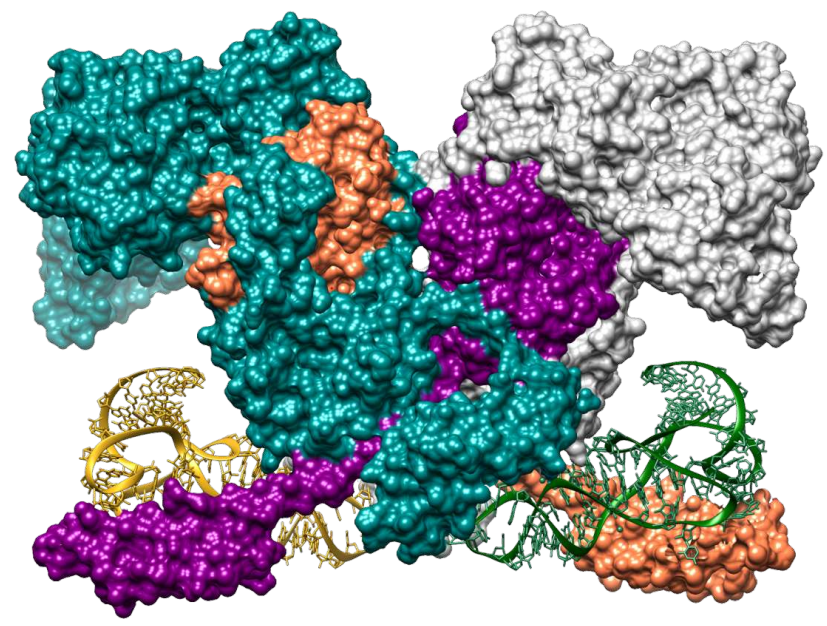

B

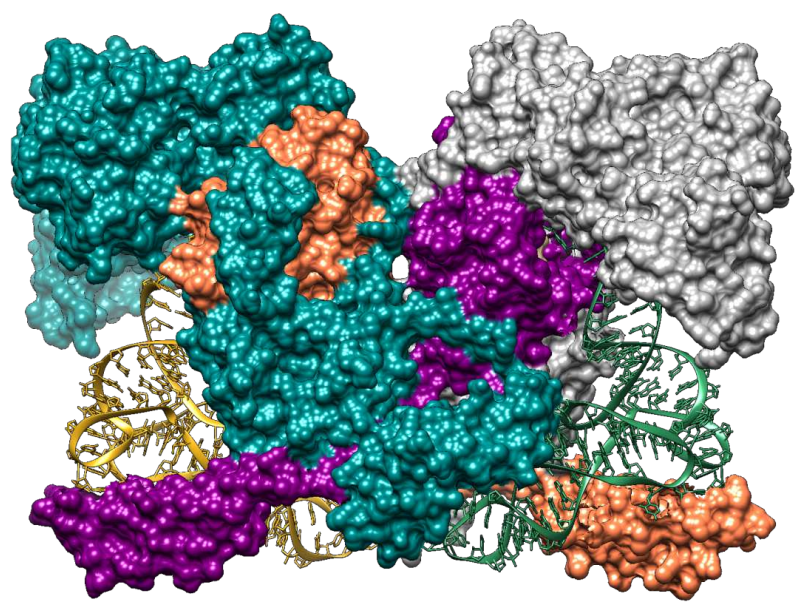


Figure 4.
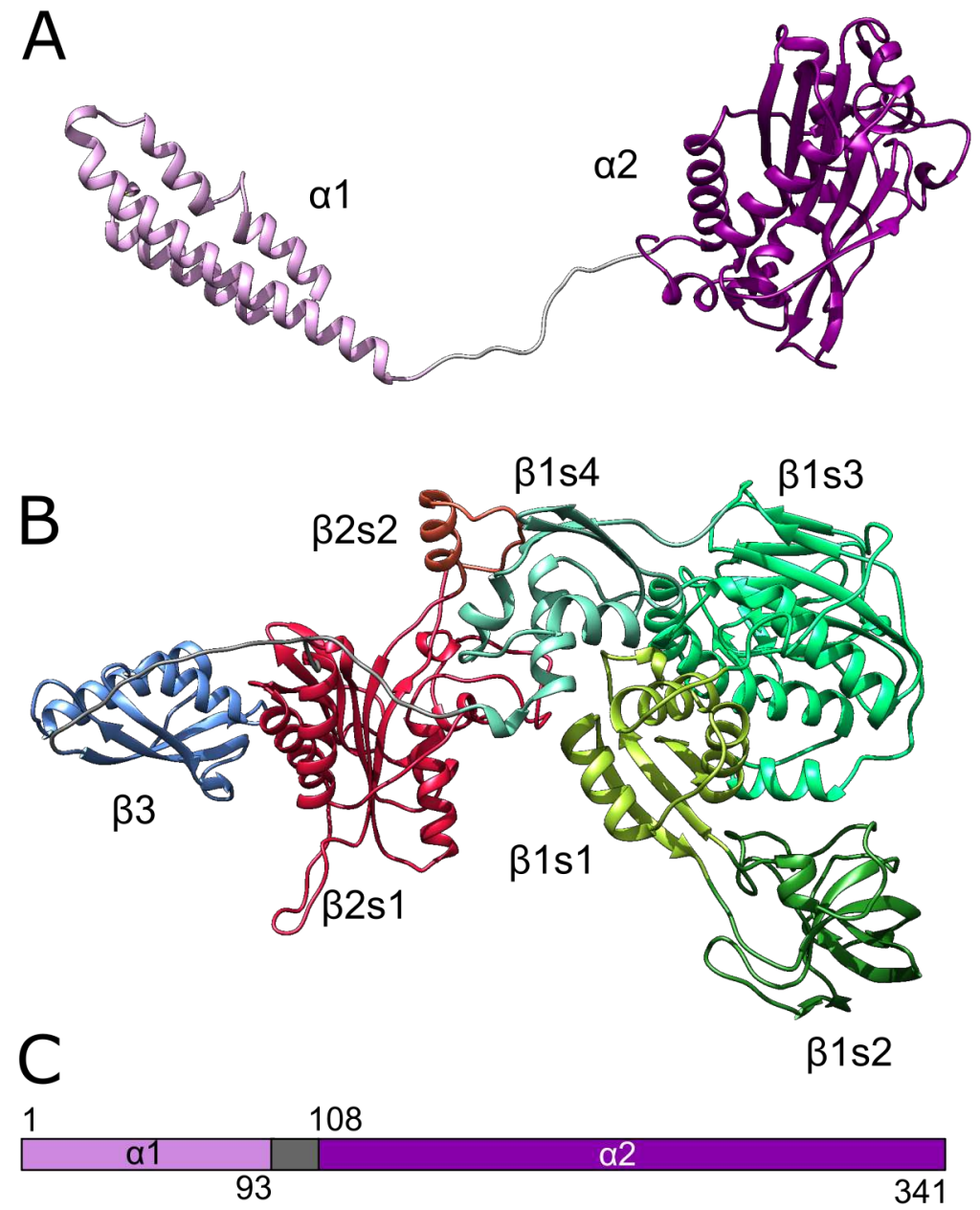

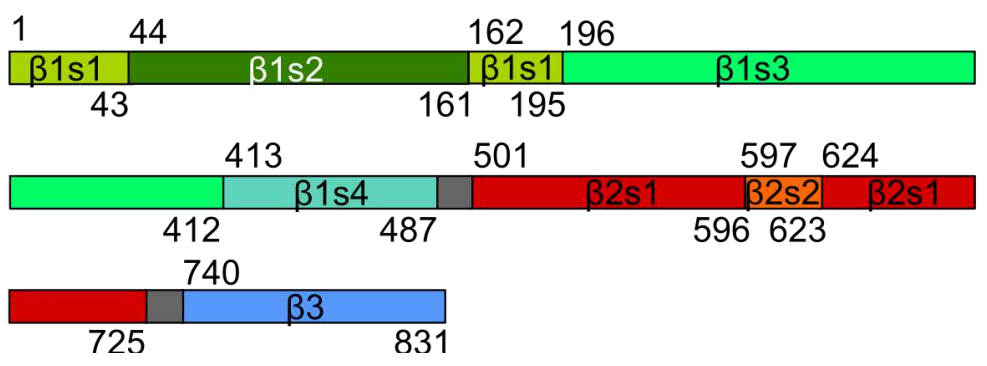


Figure 5.
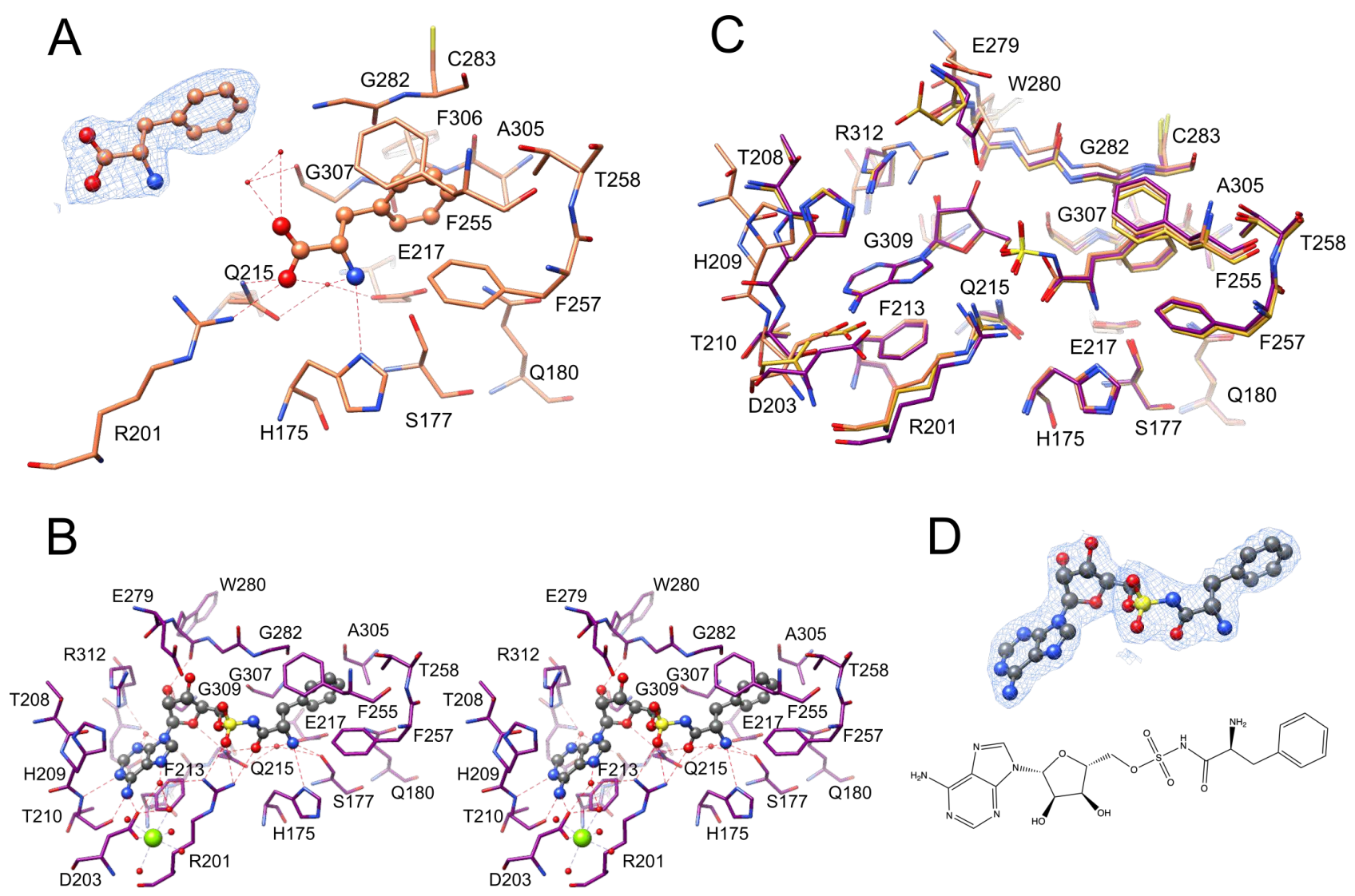
Figure 6.
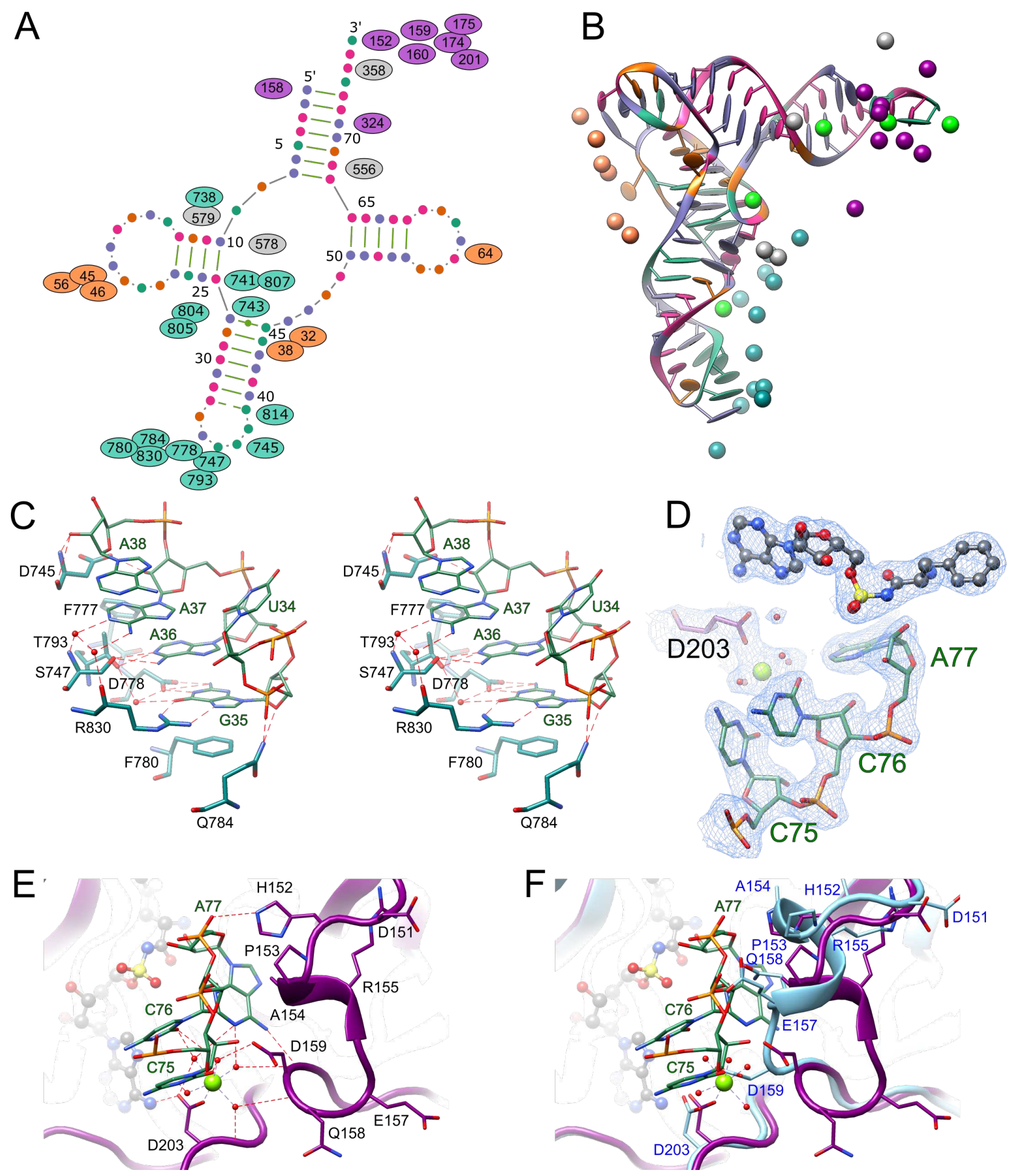
Figure 7.
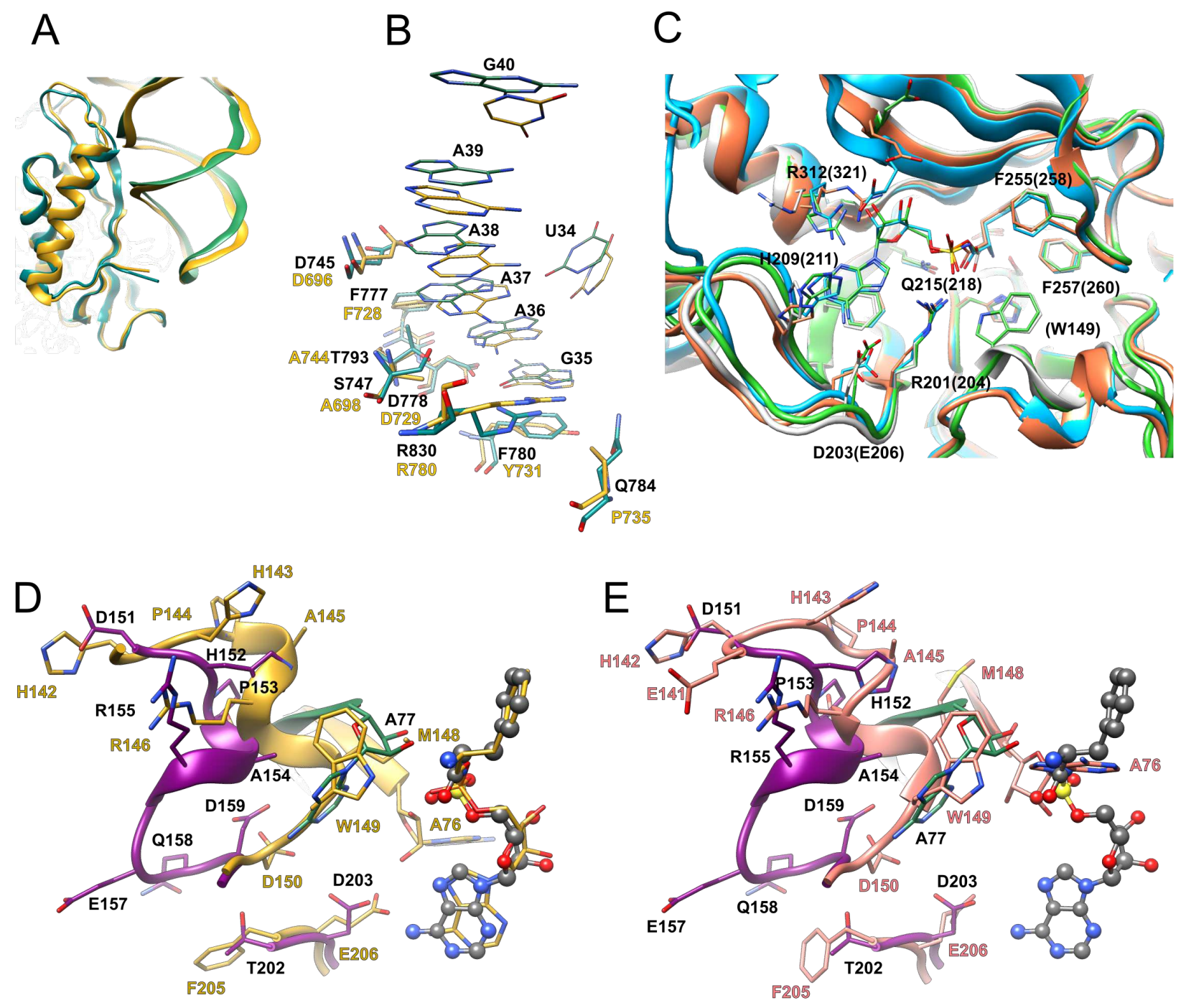

F

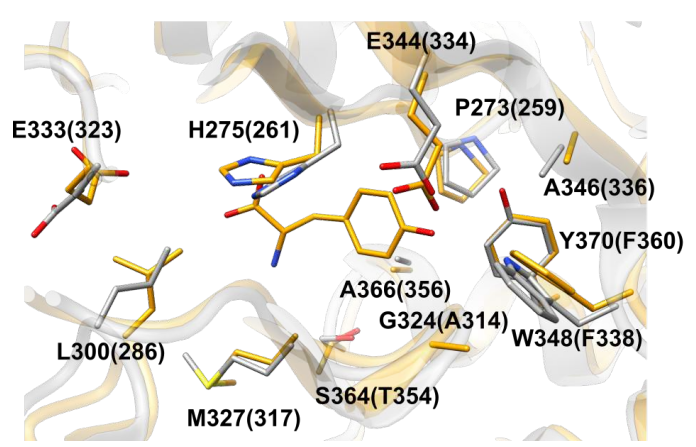


Figure 8.
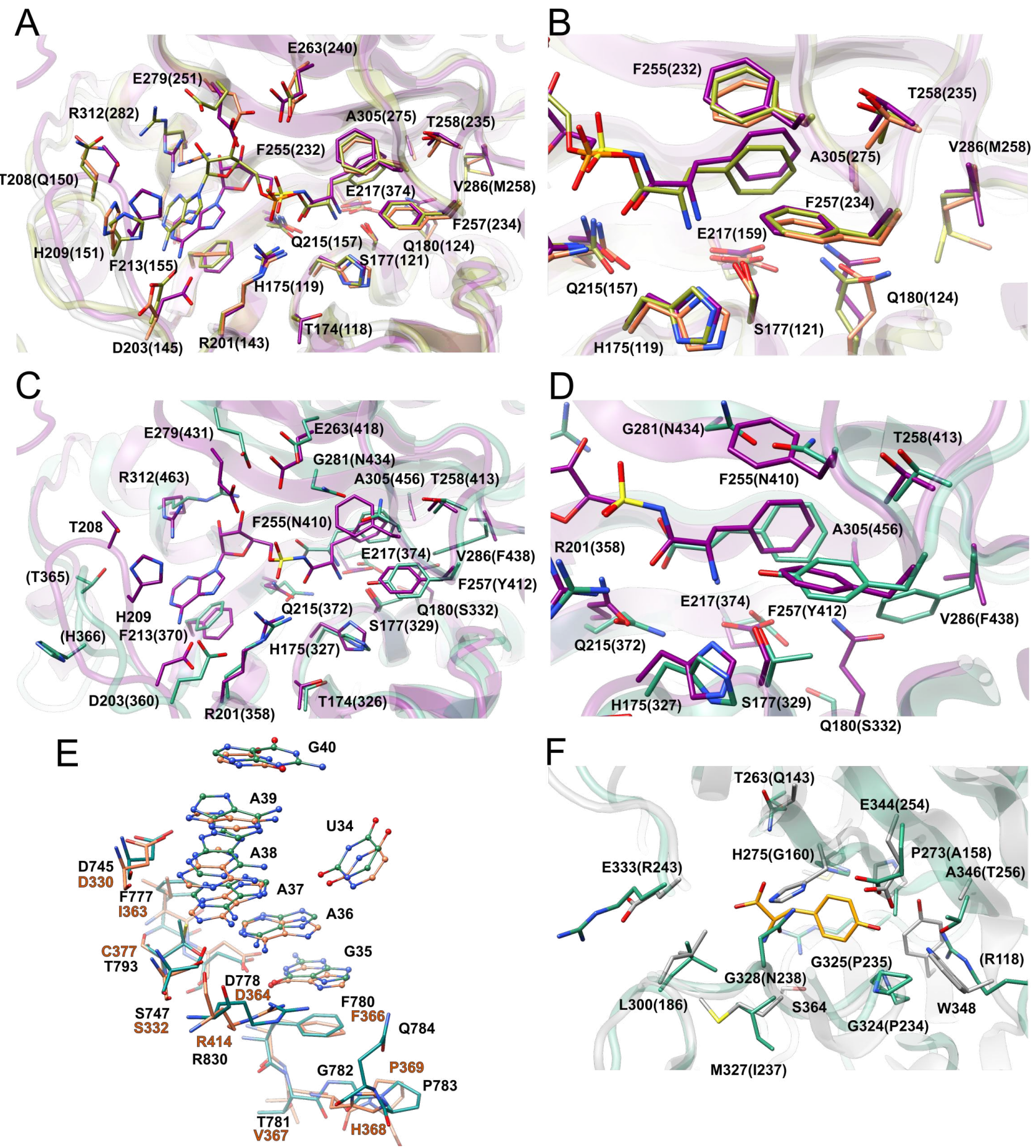


\section{Supplemental figures}

Figure S1. Sequence alignment of FRSs. (A) Subunit $\alpha$. (B) Subunit $\beta$. The species include: $M$. tuberculosis, T. thermophilus, E. coli, P. aeruginosa, S. haemolyticus, K. pneumoniae, A. baumannii, S. aureus, C. difficile, L. pneumophila, E. faecium. Crystallographic models for the biologically relevant FRS heterotetramers exist for the top 5 homologs.
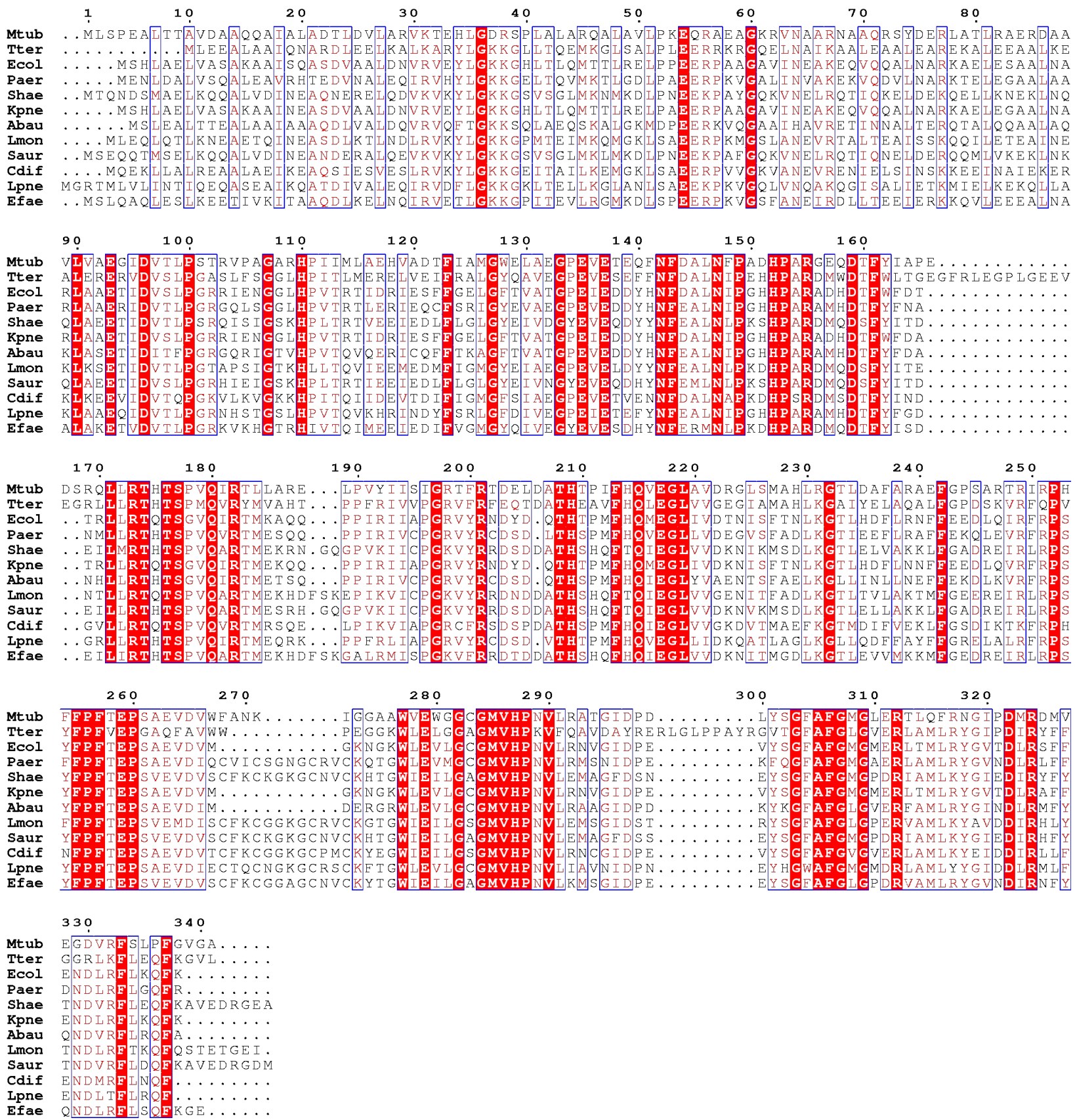

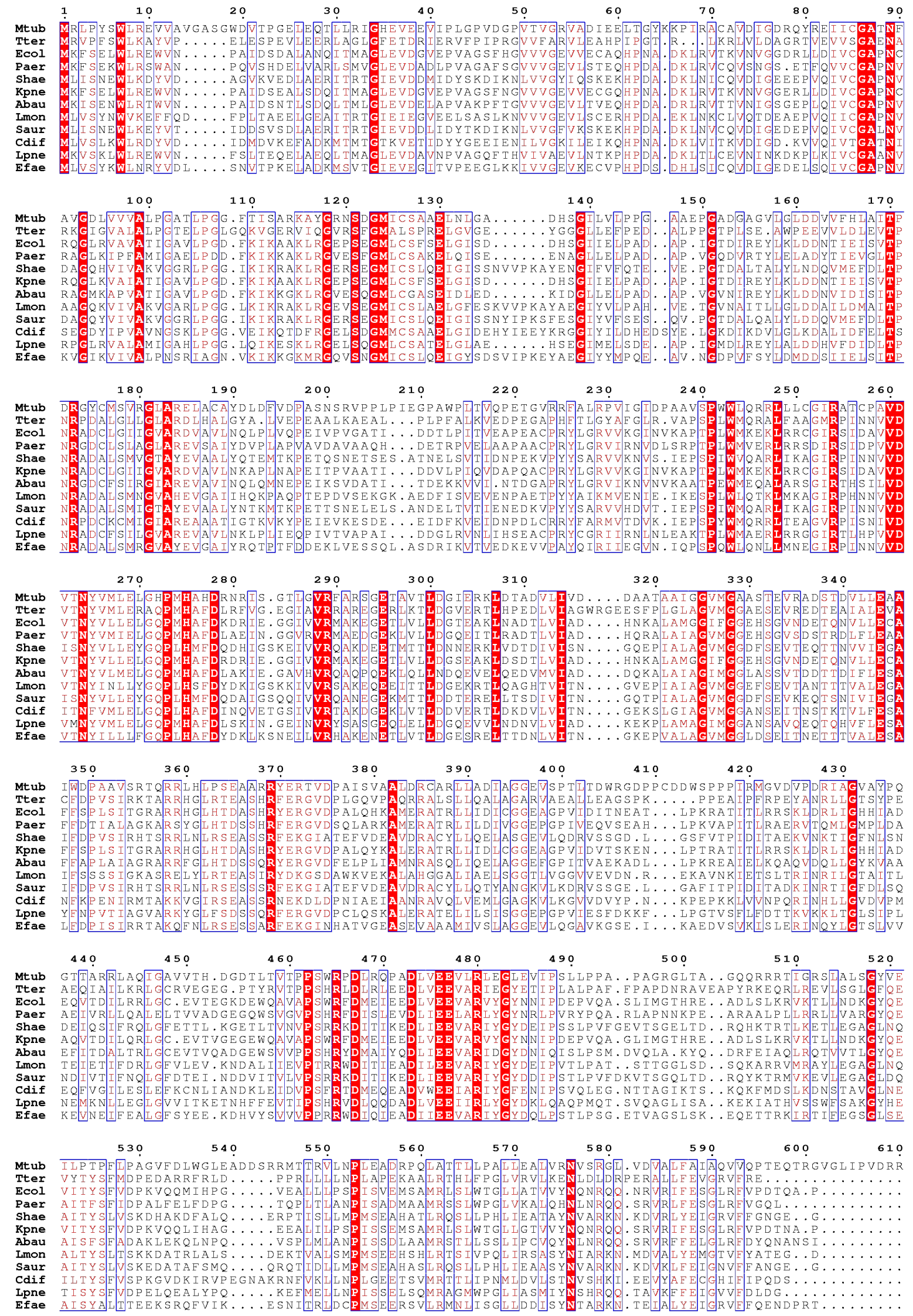
620

630

640

650

660

670

680

690

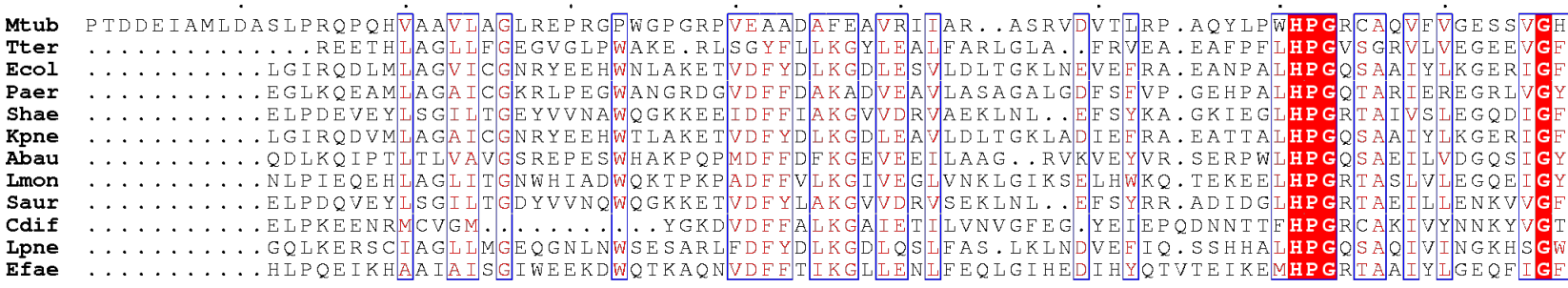

700

710

720

730

740

750

760

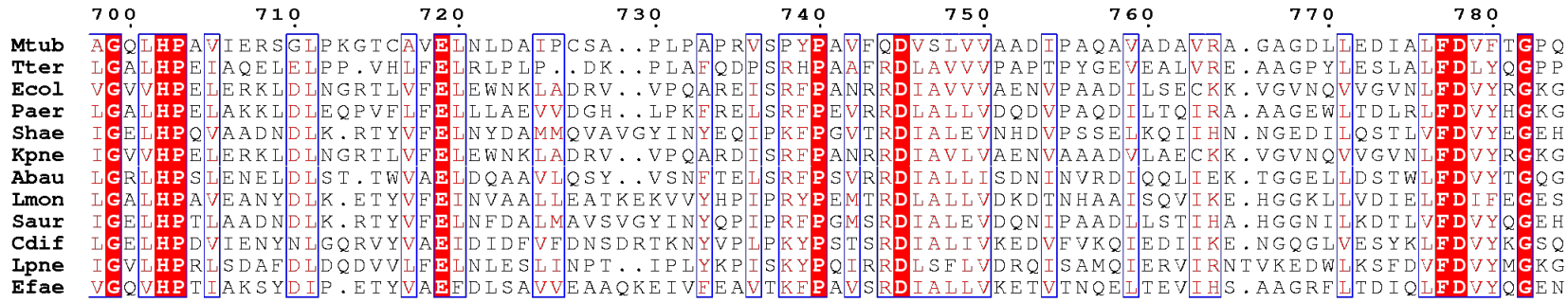

780

Efae

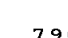

800

810

820

830

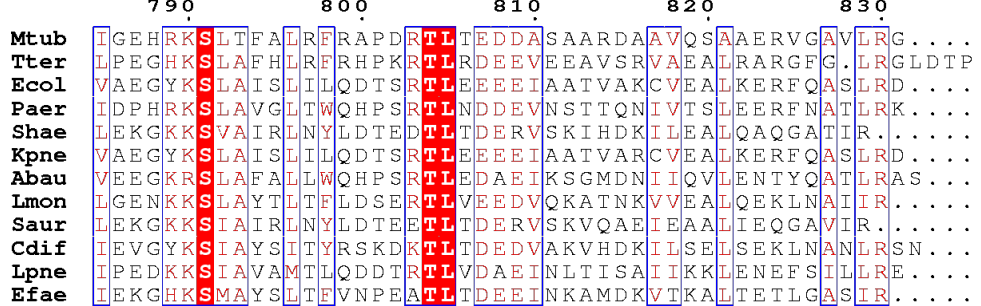




\section{Fig. S2. Domain assignment in MtFRS.}

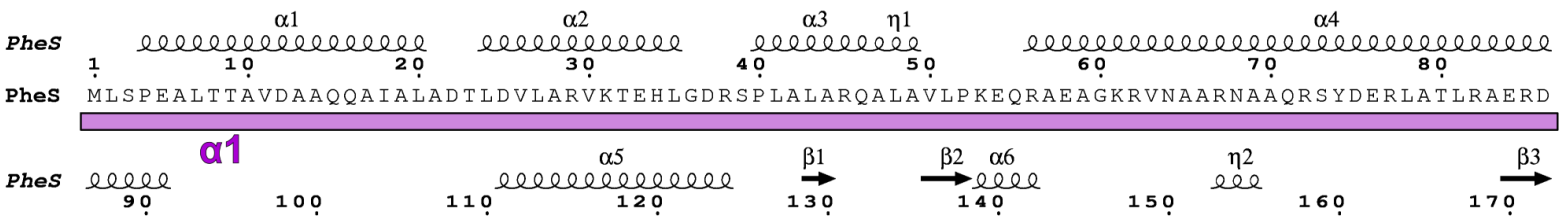
Phes AAVLVAEGIDVTLPTRVPAGARHPIMLAEHVADTFIAMGWELAEGPEVETEQFNFDALNFPADHPARGEQDTFYIAPEDSRQLL

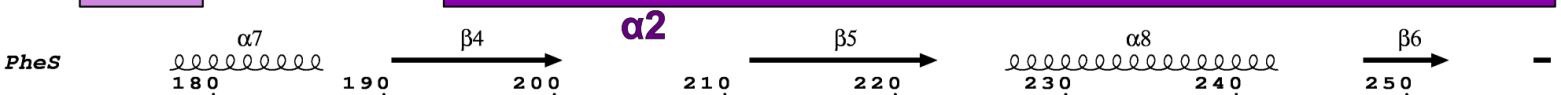

Phes RTHTSPVQIRTLLARELPVYIISIGRTFRTDELDATHTPIFHQVEGLÄDRGLSMAHLRGLDAFARAEFGSARTRIRPHFFPF

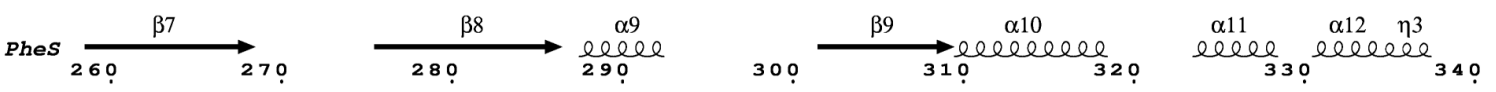

Phes EPSAEVDVWFANKIGGAAWVEWGGCGMVHPNVLRATGIDPDLYSGEAFGMGLETLQFRGIPDMRDMVEGDVRFS LPFGVGA

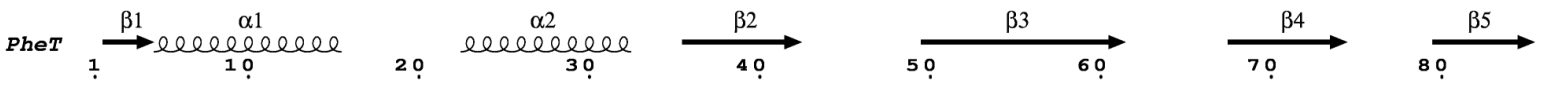

Phet MRLPYSWLREVVAVGASGWDVTPGLEQTLLRIGHEVEEVIPLGPVDGPVTVGRVADIEELTGYKKPIRACAVDIGDRQYREIICG

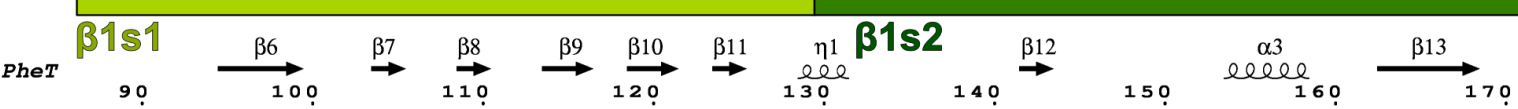

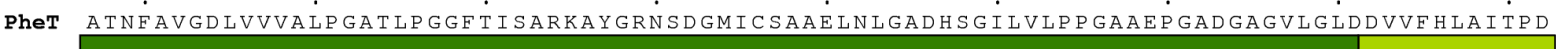

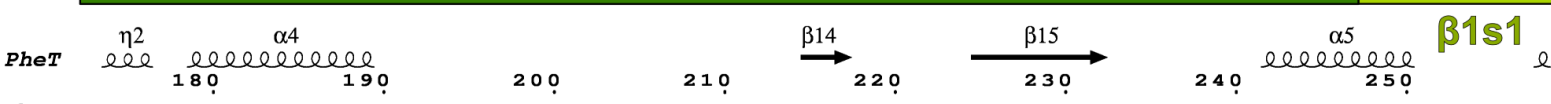

Phet RGYCMSVRGLARELACAYDLDFDPASNSRVPLPIEGPAWPLTVQPETGVRRFALRVIGIDPAAVSPWWLQRRLLCGIRATCP

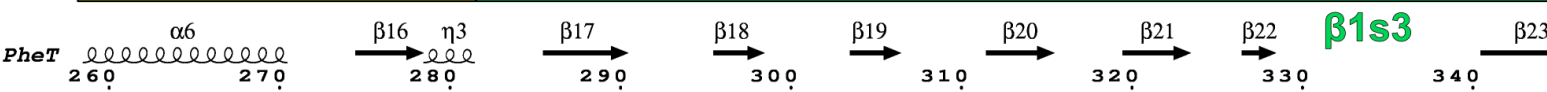

PheT AVDVTNYVMLEGHPMHAHRNRISGTLGVRFARSGETAVTLDGIERKLDTADVLIVDDAATAAIGGVMGAASTEVRADSTDVLLE

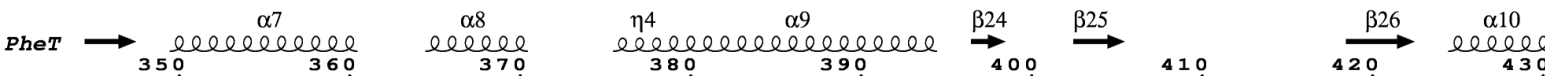

PheT AAIWDPAAVSTQRRLHLPSEAARRYRTVDPAISVAALDRCARLLADIAGGEVSPTLTDWRGDPPCDDWSPPPIRMGVDVPDRIA

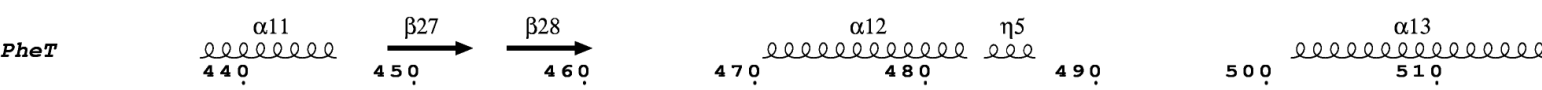

Phet GVAYPQTTARRLAQIGAVVTHDGDTLTVTPSWRPDLRQPADLVEEVLRLEGLEVIPSVLPPAPAGRGLTAGQQRRRTIGRSLAL

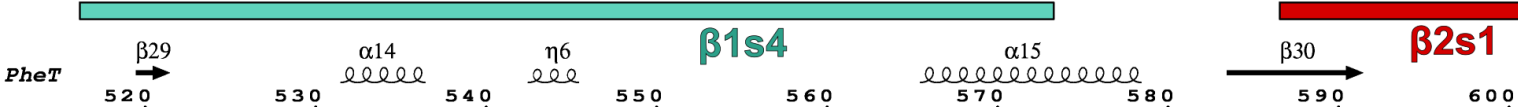

PheT SGYVEILPTPFLPAGFDLWGEADDSRRMTTRLNPLEADRPQLATTLLPALLEALVRVSRGLVDVALFAIAQVVQPTEQTRGV

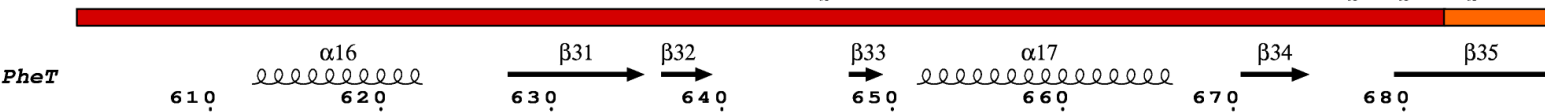

PheT GLIPVDRRPTDDEIAMLDASLPRQPQHVAAVLAGLREPRGPWGPGRPVEAADAFEAVRIIARASRVDVTLRPAQYLPWHPGRCAQV

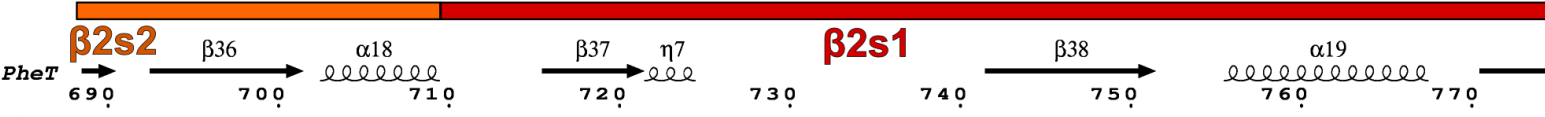

PheT FVGESSVGHAGLHPAVIERSGLPKGTCAVELNLDAIPCSAPLPAPRVSPYPAVFQDVSLVVAADIPAQAVADAVRAGAGDLLEDI

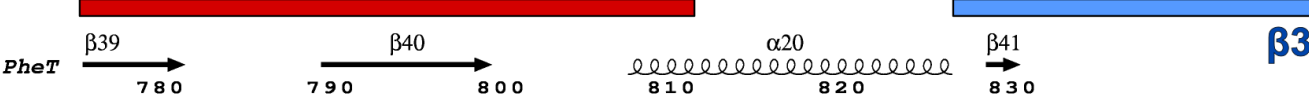

Phet ALFDVFTGPIGEHRKSLTFARFRAPDRTIEDDASAARDAAVQSAAERVGAVLRG 


\section{References}

1 World Health Organization. Global tuberculosis report 2019. (2019).

2 Mabhula, A. \& Singh, V. Drug-resistance in Mycobacterium tuberculosis: where we stand. Medchemcomm 10, 1342-1360, doi:10.1039/c9md00057g (2019).

3 Shetye, G. S., Franzblau, S. G. \& Cho, S. New tuberculosis drug targets, their inhibitors, and potential therapeutic impact. Transl Res, doi:10.1016/j.trsl.2020.03.007 (2020).

$4 \quad \mathrm{Li}, \mathrm{X}$. et al. Discovery of a Potent and Specific M. tuberculosis Leucyl-tRNA Synthetase Inhibitor: (S)-3-(Aminomethyl)-4-chloro-7-(2-hydroxyethoxy)benzo[c][1,2]oxaborol-1(3H)-ol (GSK656). J Med Chem 60, 8011-8026, doi:10.1021/acs.jmedchem.7b00631 (2017).

5 Wallis, R. S. et al. Mycobactericidal activity of sutezolid (PNU-100480) in sputum (EBA) and blood (WBA) of patients with pulmonary tuberculosis. PLoS One 9, e94462, doi:10.1371/journal.pone.0094462 (2014).

6 Ibba, M. \& Soll, D. Aminoacyl-tRNA synthesis. Annu Rev Biochem 69, 617-650, doi:10.1146/annurev.biochem.69.1.617 (2000).

7 Ibba, M. et al. Substrate recognition by class I lysyl-tRNA synthetases: a molecular basis for gene displacement. Proc Natl Acad Sci U S A 96, 418-423, doi:10.1073/pnas.96.2.418 (1999).

8 Beyer, D. et al. New class of bacterial phenylalanyl-tRNA synthetase inhibitors with high potency and broad-spectrum activity. Antimicrob Agents Chemother 48, 525-532, doi:10.1128/aac.48.2.525-532.2004 (2004).

9 Diaz-Lazcoz, Y. et al. Evolution of genes, evolution of species: the case of aminoacyl-tRNA synthetases. Mol Biol Evol 15, 1548-1561, doi:10.1093/oxfordjournals.molbev.a025882 (1998).

10 Klipcan, L. et al. Crystal structure of human mitochondrial PheRS complexed with tRNA(Phe) in the active "open" state. J Mol Biol 415, 527-537, doi:10.1016/j.jmb.2011.11.029 (2012).

11 Lee, E. Y., Kim, S. \& Kim, M. H. Aminoacyl-tRNA synthetases, therapeutic targets for infectious diseases. Biochem Pharmacol 154, 424-434, doi:10.1016/j.bcp.2018.06.009 (2018).

12 Mosyak, L., Reshetnikova, L., Goldgur, Y., Delarue, M. \& Safro, M. G. Structure of phenylalanyl-tRNA synthetase from Thermus thermophilus. Nat Struct Biol 2, 537-547, doi:10.1038/nsb0795-537 (1995).

13 Sasaki, H. M. et al. Structural and mutational studies of the amino acid-editing domain from archaeal/eukaryal phenylalanyl-tRNA synthetase. Proc Natl Acad Sci U S A 103, 14744-14749, doi:10.1073/pnas.0603182103 (2006).

14 Evdokimov, A. G. et al. Rational protein engineering in action: the first crystal structure of a phenylalanine tRNA synthetase from Staphylococcus haemolyticus. J Struct Biol 162, 152-169, doi:10.1016/j.jsb.2007.11.002 (2008).

15 Finarov, I., Moor, N., Kessler, N., Klipcan, L. \& Safro, M. G. Structure of human cytosolic phenylalanyl-tRNA synthetase: evidence for kingdom-specific design of the active sites and tRNA binding patterns. Structure 18, 343-353, doi:10.1016/j.str.2010.01.002 (2010).

16 Mermershtain, I. et al. Idiosyncrasy and identity in the prokaryotic Phe-system: crystal structure of E. coli phenylalanyl-tRNA synthetase complexed with phenylalanine and AMP. Protein science : a publication of the Protein Society 20, 160-167, doi:10.1002/pro.549 (2011). 17 Reshetnikova, L., Moor, N., Lavrik, O. \& Vassylyev, D. G. Crystal structures of phenylalanyl-tRNA synthetase complexed with phenylalanine and a phenylalanyl-adenylate analogue. J Mol Biol 287, 555-568, doi:10.1006/jmbi.1999.2617 (1999). 
18 Kotik-Kogan, O., Moor, N., Tworowski, D. \& Safro, M. Structural basis for discrimination of L-phenylalanine from L-tyrosine by phenylalanyl-tRNA synthetase. Structure 13, 1799-1807, doi:10.1016/j.str.2005.08.013 (2005).

19 Ho, J. M., Bakkalbasi, E., Soll, D. \& Miller, C. A. Drugging tRNA aminoacylation. RNA Biol 15, 667-677, doi:10.1080/15476286.2018.1429879 (2018).

20 Kwon, N. H., Fox, P. L. \& Kim, S. Aminoacyl-tRNA synthetases as therapeutic targets. Nat Rev Drug Discov 18, 629-650, doi:10.1038/s41573-019-0026-3 (2019).

21 Jarvest, R. L. et al. Discovery and optimisation of potent, selective, ethanolamine inhibitors of bacterial phenylalanyl tRNA synthetase. Bioorg Med Chem Lett 15, 2305-2309, doi:10.1016/j.bmcl.2005.03.003 (2005).

$22 \mathrm{Hu}$, Y., Palmer, S. O., Munoz, H. \& Bullard, J. M. High Throughput Screen Identifies Natural Product Inhibitor of Phenylalanyl-tRNA Synthetase from Pseudomonas aeruginosa and Streptococcus pneumoniae. Curr Drug Discov Technol 11, 279-292, doi:10.2174/1570163812666150120154701 (2014).

23 Wang, L.-N. et al. Small-molecule inhibitors of the tuberculosis target, phenylalanyl-tRNA synthetase from Penicillium griseofulvum CPCC-400528. Cogent Chemistry 2, 1181536, doi:10.1080/23312009.2016.1181536 (2016).

24 Krutyholowa, R., Zakrzewski, K. \& Glatt, S. Charging the code - tRNA modification complexes. Curr Opin Struct Biol 55, 138-146, doi:10.1016/j.sbi.2019.03.014 (2019).

25 de Crecy-Lagard, V.\& Jaroch, M. Functions of Bacterial tRNA Modifications: From Ubiquity to Diversity. Trends Microbiol, doi:10.1016/j.tim.2020.06.010 (2020).

26 Yanas, A. \& Liu, K. F. RNA modifications and the link to human disease. Methods Enzymol 626, 133-146, doi:10.1016/bs.mie.2019.08.003 (2019).

27 Bernier, S. et al. Glutamylsulfamoyladenosine and pyroglutamylsulfamoyladenosine are competitive inhibitors of E. coli glutamyl-tRNA synthetase. J Enzyme Inhib Med Chem 20, 61-67, doi:10.1080/14756360400002007 (2005).

28 Forrest, A. K. et al. Aminoalkyl adenylate and aminoacyl sulfamate intermediate analogues differing greatly in affinity for their cognate Staphylococcus aureus aminoacyl tRNA synthetases. Bioorg Med Chem Lett 10, 1871-1874, doi:10.1016/s0960-894x(00)00360-7 (2000).

29 Rath, V. L., Silvian, L. F., Beijer, B., Sproat, B. S. \& Steitz, T. A. How glutaminyl-tRNA synthetase selects glutamine. Structure 6, 439-449, doi:10.1016/s0969-2126(98)00046-x (1998). 30 Eriani, G., Delarue, M., Poch, O., Gangloff, J. \& Moras, D. Partition of tRNA synthetases into two classes based on mutually exclusive sets of sequence motifs. Nature 347, 203-206, doi:10.1038/347203a0 (1990).

31 Loftfield, R. B., Eigner, E. A. \& Pastuszyn, A. The role of spermine in preventing misacylation by phenylalanyl-tRNA synthetase. J Biol Chem 256, 6729-6735 (1981).

32 Kim, Y., Opron, K. \& Burton, Z. F. A tRNA- and Anticodon-Centric View of the Evolution of Aminoacyl-tRNA Synthetases, tRNAomes, and the Genetic Code. Life (Basel) 9 , doi:10.3390/life9020037 (2019).

33 Carter, C. W., Jr. Urzymology: experimental access to a key transition in the appearance of enzymes. J Biol Chem 289, 30213-30220, doi:10.1074/jbc.R114.567495 (2014).

34 Carter, C. W., Jr. \& Wills, P. R. Experimental solutions to problems defining the origin of codon-directed protein synthesis. Biosystems 183, 103979, doi:10.1016/j.biosystems.2019.103979 (2019). 
35 Schimmel, P., Giege, R., Moras, D. \& Yokoyama, S. An operational RNA code for amino acids and possible relationship to genetic code. Proc Natl Acad Sci U S A 90, 8763-8768, doi:10.1073/pnas.90.19.8763 (1993).

36 Sampson, J. R., Behlen, L. S., DiRenzo, A. B. \& Uhlenbeck, O. C. Recognition of yeast tRNA(Phe) by its cognate yeast phenylalanyl-tRNA synthetase: an analysis of specificity.

Biochemistry 31, 4161-4167, doi:10.1021/bi00132a002 (1992).

37 Kim, Y. et al. High-throughput protein purification and quality assessment for crystallization. Methods 55, 12-28, doi:10.1016/j.ymeth.2011.07.010 (2011).

38 Sherlin, L. D. et al. Chemical and enzymatic synthesis of tRNAs for high-throughput crystallization. RNA 7, 1671-1678 (2001).

39 Wellington, S. et al. A small-molecule allosteric inhibitor of Mycobacterium tuberculosis tryptophan synthase. Nat. Chem. Biol. 13, 943-950, doi:10.1038/nchembio.2420 (2017).

40 Minor, W., Cymborowski, M., Otwinowski, Z. \& Chruszcz, M. HKL-3000: the integration of data reduction and structure solution--from diffraction images to an initial model in minutes.

Acta Crystallographica Section D-Biological Crystallography 62, 859-866, doi:10.1107/S0907444906019949 (2006).

41 French, S. W., K. On the Treatment of Negative Intensity Observations Acta Cryst. A34, 517-525, doi:10.1107/S0567739478001114 (1977).

42 Padilla, J. E. \& Yeates, T. O. A statistic for local intensity differences: robustness to anisotropy and pseudo-centering and utility for detecting twinning. Acta Crystallographica Section D-Biological Crystallography 59, 1124-1130 (2003).

43 Winn, M. D. et al. Overview of the CCP4 suite and current developments. Acta

Crystallographica Section D-Biological Crystallography 67, 235-242, doi:S0907444910045749

[pii]

10.1107/S0907444910045749 (2011).

44 Emsley, P.\& Cowtan, K. Coot: model-building tools for molecular graphics. Acta

Crystallographica Section D-Biological Crystallography 60, 2126-2132,

doi:10.1107/S0907444904019158 (2004).

45 Winn, M. D., Murshudov, G. N. \& Papiz, M. Z. Macromolecular TLS refinement in

REFMAC at moderate resolutions. Methods Enzymol 374, 300-321, doi:10.1016/S0076-

6879(03)74014-2

S0076687903740142 [pii] (2003).

46 Afonine, P. V.et al. Towards automated crystallographic structure refinement with

phenix.refine. Acta Crystallogr., Sect D: Biol. Crystallogr. 68, 352-367,

doi:10.1107/S0907444912001308 (2012).

47 Huang, C. C. et al. Ucsf Chimera. Abstr Pap Am Chem S 238 (2009).

48 Gong, S., Zhang, C. \& Zhang, Y. RNA-align: quick and accurate alignment of RNA 3D

structures based on size-independent TM-scoreRNA. Bioinformatics 35, 4459-4461, doi:10.1093/bioinformatics/btz282 (2019).

49 Wower, I. K., Zwieb, C. W., Guven, S. A. \& Wower, J. Binding and cross-linking of tmRNA to ribosomal protein $\mathrm{S} 1$, on and off the Escherichia coli ribosome. EMBO J. 19, 6612-6621, doi:10.1093/emboj/19.23.6612 (2000).

50 Varshney, U., Lee, C. P. \& RajBhandary, U. L. Direct analysis of aminoacylation levels of tRNAs in vivo. Application to studying recognition of Escherichia coli initiator tRNA mutants by glutaminyl-tRNA synthetase. J Biol Chem 266, 24712-24718 (1991). 
51 Cheng, Y. \& Prusoff, W. H. Relationship between the inhibition constant (K1) and the concentration of inhibitor which causes 50 per cent inhibition (I50) of an enzymatic reaction. Biochem Pharmacol 22, 3099-3108, doi:10.1016/0006-2952(73)90196-2 (1973).

52 Bullock, T. L., Uter, N., Nissan, T. A. \& Perona, J. J. Amino acid discrimination by a class I aminoacyl-tRNA synthetase specified by negative determinants. J Mol Biol 328, 395-408, doi:10.1016/s0022-2836(03)00305-x (2003).

53 Ishikawa, F. \& Kakeya, H. Specific enrichment of nonribosomal peptide synthetase module by an affinity probe for adenylation domains. Bioorg Med Chem Lett 24, 865-869, doi:10.1016/j.bmcl.2013.12.082 (2014).

54 Goldgur, Y. et al. The crystal structure of phenylalanyl-tRNA synthetase from thermus thermophilus complexed with cognate tRNAPhe. Structure 5, 59-68, doi:10.1016/s09692126(97)00166-4 (1997).

55 Fishman, R., Ankilova, V., Moor, N. \& Safro, M. Structure at 2.6 A resolution of phenylalanyl-tRNA synthetase complexed with phenylalanyl-adenylate in the presence of manganese. Acta Crystallogr D Biol Crystallogr 57, 1534-1544, doi:10.1107/s090744490101321x (2001).

56 Moor, N., Kotik-Kogan, O., Tworowski, D., Sukhanova, M. \& Safro, M. The crystal structure of the ternary complex of phenylalanyl-tRNA synthetase with tRNAPhe and a phenylalanyl-adenylate analogue reveals a conformational switch of the CCA end. Biochemistry $\mathbf{4 5}$, 10572-10583, doi:10.1021/bi0604911 (2006).

57 Klipcan, L., Moor, N., Kessler, N. \& Safro, M. G. Eukaryotic cytosolic and mitochondrial phenylalanyl-tRNA synthetases catalyze the charging of tRNA with the meta-tyrosine. Proc Natl Acad Sci U S A 106, 11045-11048, doi:10.1073/pnas.0905212106 (2009).

58 Moor, N., Klipcan, L. \& Safro, M. G. Bacterial and eukaryotic phenylalanyl-tRNA synthetases catalyze misaminoacylation of tRNA(Phe) with 3,4-dihydroxy-L-phenylalanine. Chem Biol 18, 1221-1229, doi:10.1016/j.chembiol.2011.08.008 (2011).

59 Tworowski, D., Klipcan, L., Peretz, M., Moor, N. \& Safro, M. G. Universal pathway for posttransfer editing reactions: insights from the crystal structure of TtPheRS with puromycin. Proc Natl Acad Sci U S A 112, 3967-3972, doi:10.1073/pnas.1414852112 (2015).

60 Abibi, A. et al. The role of a novel auxiliary pocket in bacterial phenylalanyl-tRNA synthetase druggability. J Biol Chem 289, 21651-21662, doi:10.1074/jbc.M114.574061 (2014). 61 Klipcan, L. et al. The tRNA-induced conformational activation of human mitochondrial phenylalanyl-tRNA synthetase. Structure 16, 1095-1104, doi:10.1016/j.str.2008.03.020 (2008). 62 Kartvelishvili, E. et al. Kinetic and structural changes in HsmtPheRS, induced by pathogenic mutations in human FARS2. Protein science : a publication of the Protein Society 26, 1505-1516, doi:10.1002/pro.3176 (2017).

63 Needleman, S. B. \& Wunsch, C. D. A general method applicable to the search for similarities in the amino acid sequence of two proteins. $J$ Mol Biol 48, 443-453, doi:10.1016/00222836(70)90057-4 (1970).

64 Karplus, P. A. \& Diederichs, K. Linking crystallographic model and data quality. Science 336, 1030-1033, doi:10.1126/science.1218231 (2012).

65 Davis, I. W., Murray, L. W., Richardson, J. S. \& Richardson, D. C. MOLPROBITY: structure validation and all-atom contact analysis for nucleic acids and their complexes. Nucleic Acids Res. 32, W615-619, doi:10.1093/nar/gkh398 32/suppl_2/W615 [pii] (2004). 


\section{Figures}

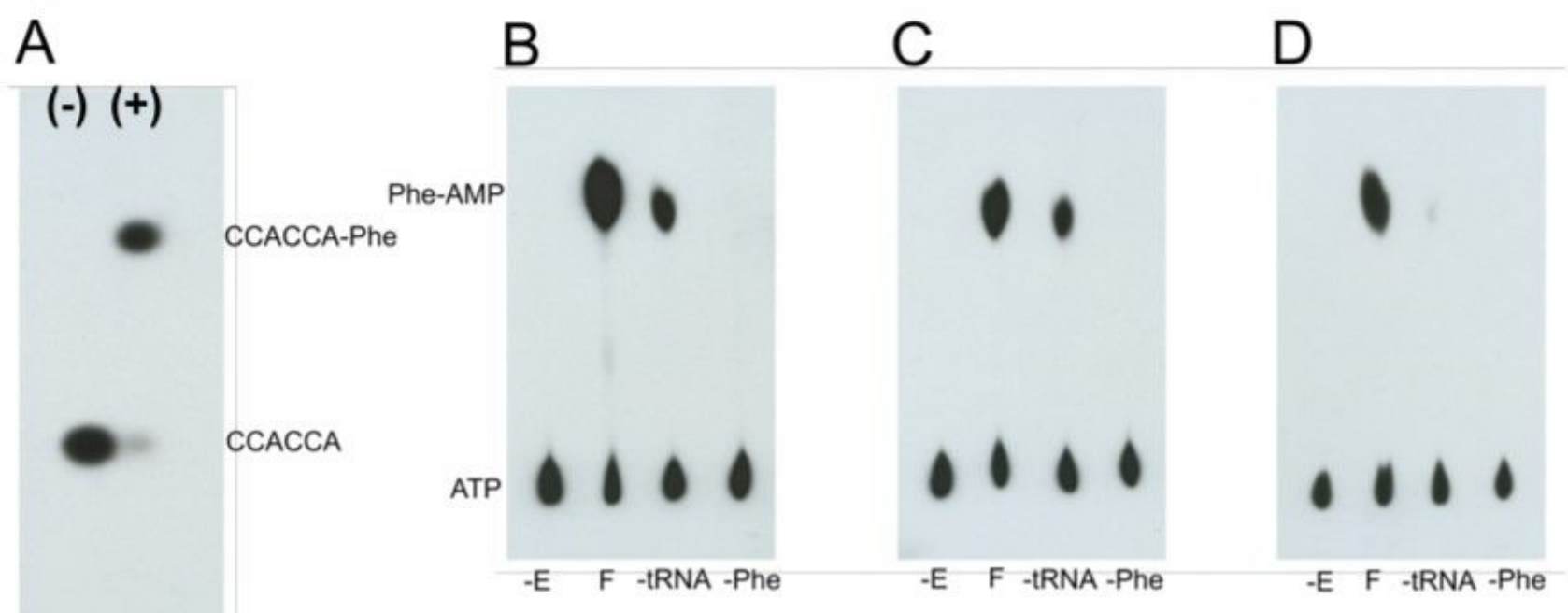

\section{Figure 1}

MtFRS activity. (A) Aminoacylation of unmodified M. tuberculosis tRNAPhe. The 3'- 32Plabeled tRNA transcript was charged with L-Phe in the presence of purified MtFRS. The extent of aminoacylation was analyzed according to Varshney et al. 50. Prior to polyacrylamide gel electrophoresis, tRNA and Phe-tRNA were digested with RNase T1 to yield 3'-32P-labeled CCACCA and CCACCA-Phe, respectively. (+) and (-) indicate the presence and the absence of the enzyme. (BD) Synthesis of the Phe-AMP intermediate by MtFRS in the presence of unmodified M. tuberculosis tRNAPhe (B), E. coli tRNAPhe (C) and yeast tRNAPhe (D). Phe-[32P]AMP synthesis was monitored by thin layer chromatography. Phe-AMP and ATP denote 32P-labeled compounds. F - full reaction; -E, -tRNA and -Phe - control reactions lacking the enzyme, tRNA or amino acid. 
A

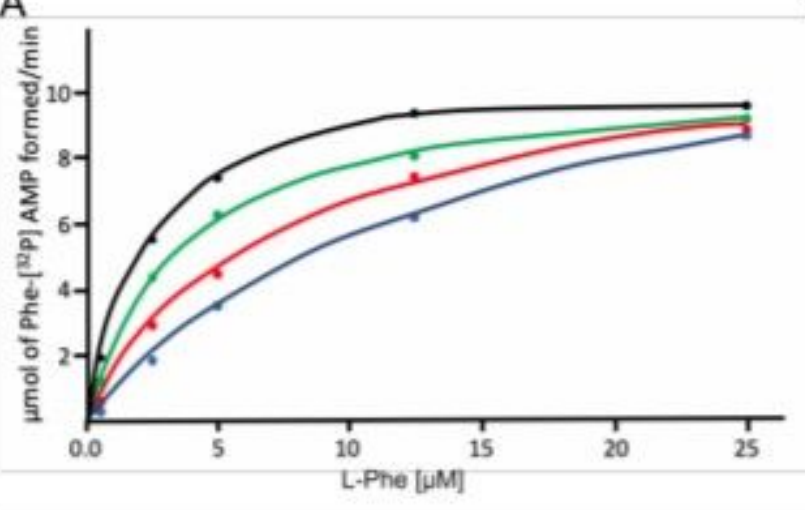

B
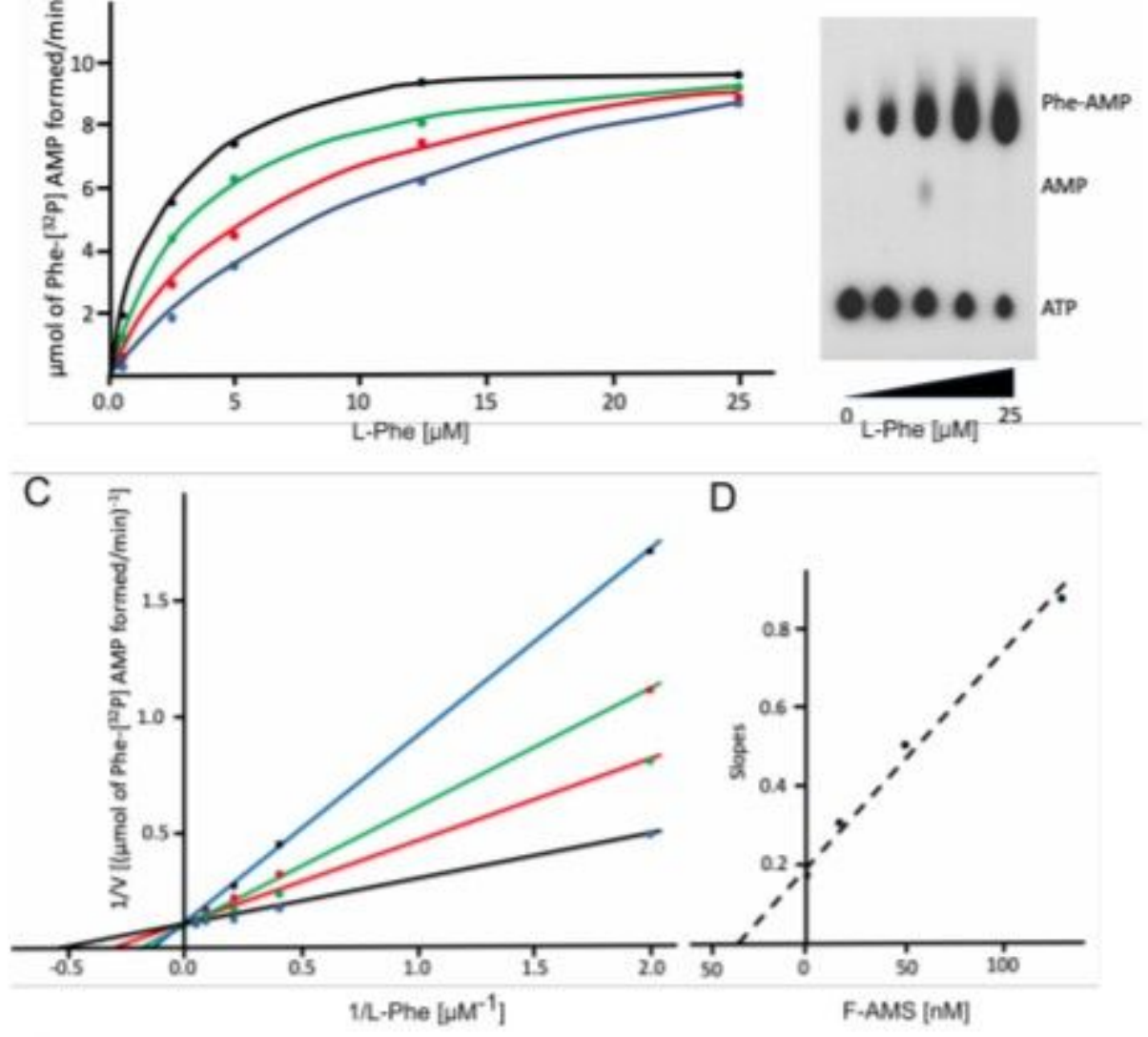

D

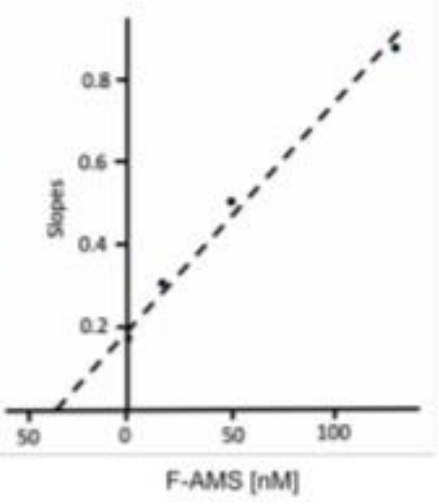

$E$

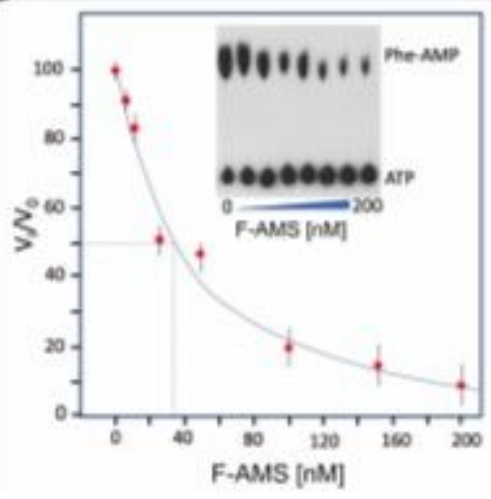

Figure 2

Inhibition of MtFRS by F-AMS. (A) Competitive binding of inhibitor with respect to LPhe. Inhibitor concentrations were as follows: $0 \mu \mathrm{M}$ (black line), $15 \mathrm{nM}$ (green line), $50 \mathrm{nM}$ (red line), and $125 \mathrm{nM}$. (B) Phe-[ 32P]AMP synthesis in the presence of $15 \mathrm{nM}$ inhibitor (corresponding to green line) monitored by thin layer chromatography. The rate of Phe-[32P]AMP formation was determined at $37^{\circ} \mathrm{C}$ over $10 \mathrm{~min}$. (CD) Competitive inhibition of MtFRS by the F-AMS shown in the form of a Lineweaver-Burk plot. Km for Phe is $1.78 \mu \mathrm{M}(\mathrm{C})$. The secondary diagram was used to determine the inhibition constant Ki, which is 33 $\mathrm{nM}$ (D). (E) Plot of the dose-response curve shows the percentage of initial velocity reduced by various concentrations of the F-AMS. The insert illustrates the inhibition of Phe-[32P]AMP synthesis monitored 
by thin layer chromatography. Dotted lines indicate that the inhibitor has IC50 value of approximately 36 $\mathrm{nM}$.
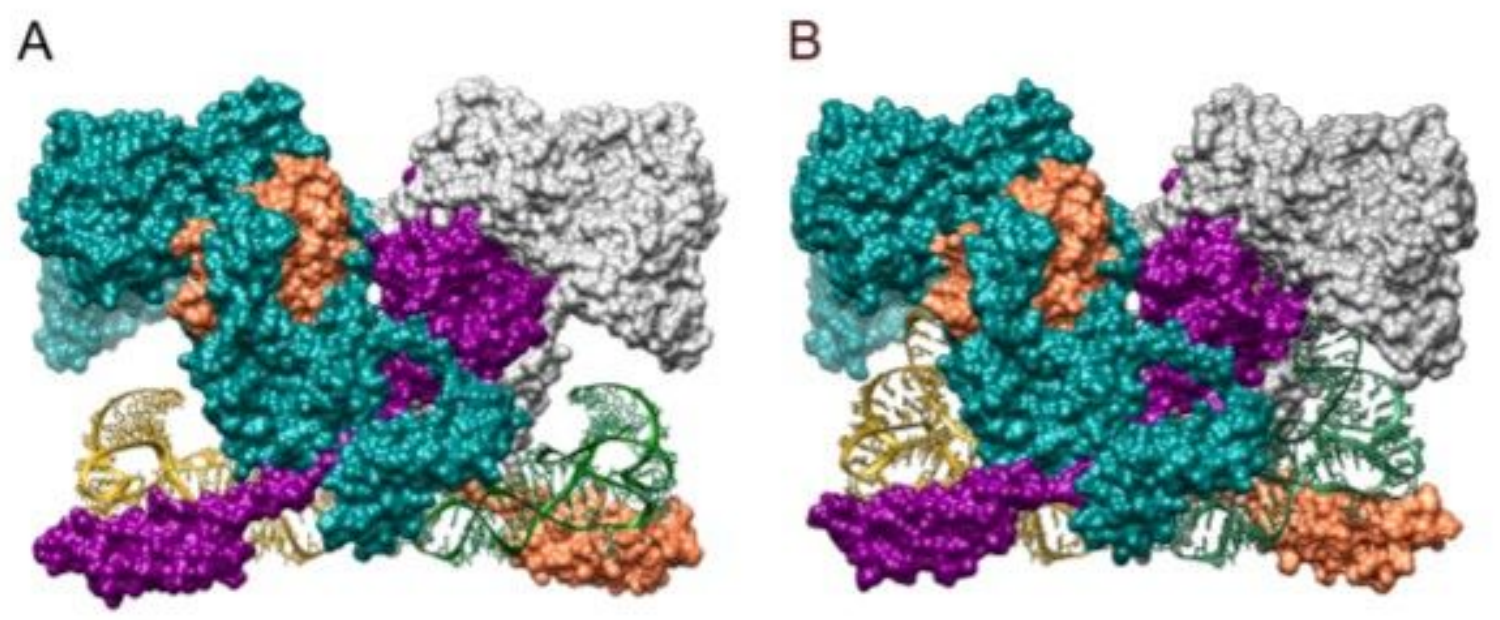

\section{Figure 3}

Overall structure of the MtFRS-tRNAPhe complex. (A) MtFRS-tRNAPhe heterotetramer with initial tRNA binding (MtFRS/F1) (B) MtFRS-tRNAPhe heterotetramer with productive tRNA binding (MtFRS/F-AMS2). Subunits a shown in purple and coral, subunits b shown in grey and teal, tRNA represented as yellow and green ribbon models. 


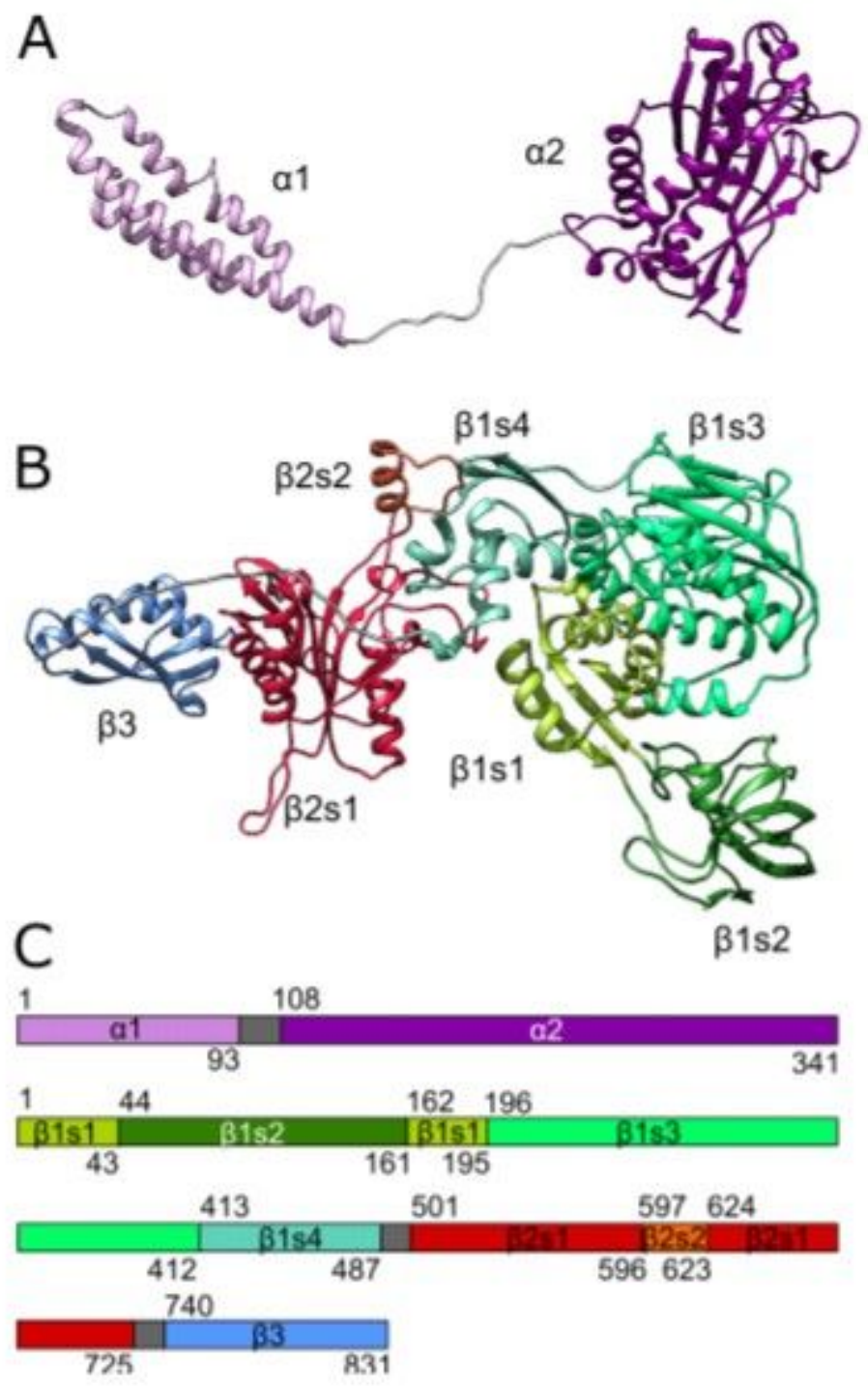

Figure 4

MtFRS domains. (A) Subunit a. (B) Subunit b. (C) Domain boundaries with starting residues numbered above the sequence-representing bars and ending residues shown under the bars. Grey sections depict linkers. 

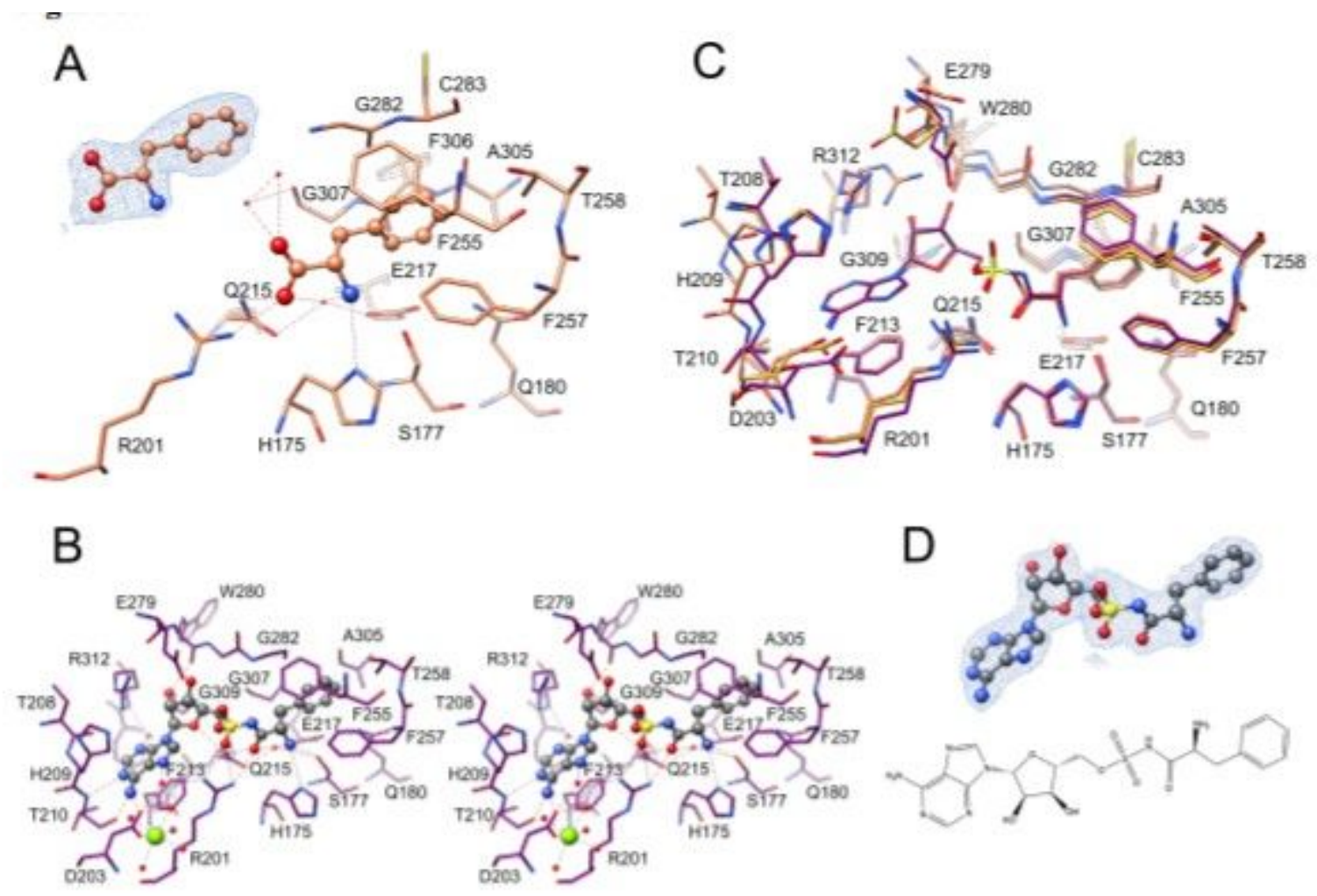

Figure 5

MtFRS interactions with ligands. (A) L-Phe binding to the aminoacylation site of FRSt/F1. The upper-left insert shows $2 \mathrm{mFo}$-DFc electron density map contoured around L-Phe at 1.2s. (B) Stereoview of F-AMS binding to the aminoacylation site of FRSt/F-AMS2. (C) Superposition of the aminoacylation site of FRSt/F-AMS2 (purple), FRSt/F-AMS1 (yellow), and FRSt/F1 (coral). (D) F-AMS ligand and its 2mFo-DFc electron density map from the FRSt/F-AMS2 complex contoured at 1.2s. Red spheres represent water molecules, a green sphere indicates magnesium ion. Hydrogen bonds are shown are dashed lines. 


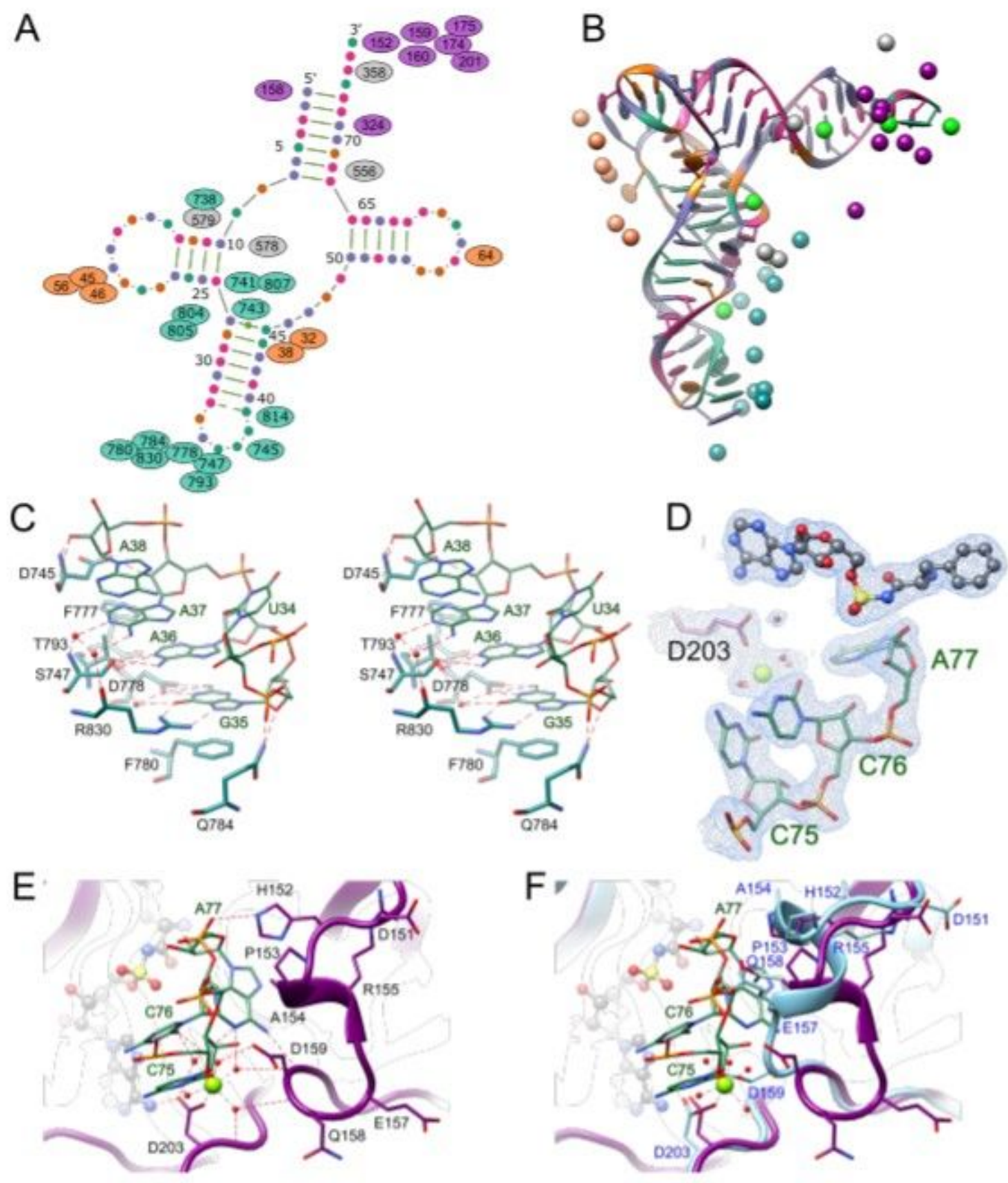

Figure 6

MtFRS interactions with tRNA. (A) tRNA molecule and interacting residues in MtFRSt/F-AMS2. (B) (B) tRNA molecule and its contacts with the polypeptide chain as seen in MtFRS/F-AMS, (chain C). The interacting residues include those forming hydrogen bonds, participating in base/amino acid pairs and base/amino acid stacks, as defined by the SNAP program (http://forum.x3dna.org/dna-proteininteractions/). The $\mathrm{Ca}$ atoms of the interacting residues are shown, colored as in $\mathrm{A}$. Magnesium ions are colored green. Nucleotides have the following colorcoding: G-purple, C-pink, A-green, U-orange. (C) Stereoview of MtFRS interactions with the anticodon loop. (D) F-AMS (grey), tRNA 3' end (green) and 
magnesium ion (green sphere) binding in the MtFRSt/F-AMS2 active site shown in 2DFo-Fc electron density map contoured at 1.2s. (E) tRNA 3' end interactions in MtFRSt/F-AMS2. (F) Superposition of the tRNA 3' end binding region of MtFRSt/F-AMS2 (purple) and MtFRSt/F-AMS1 (blue).
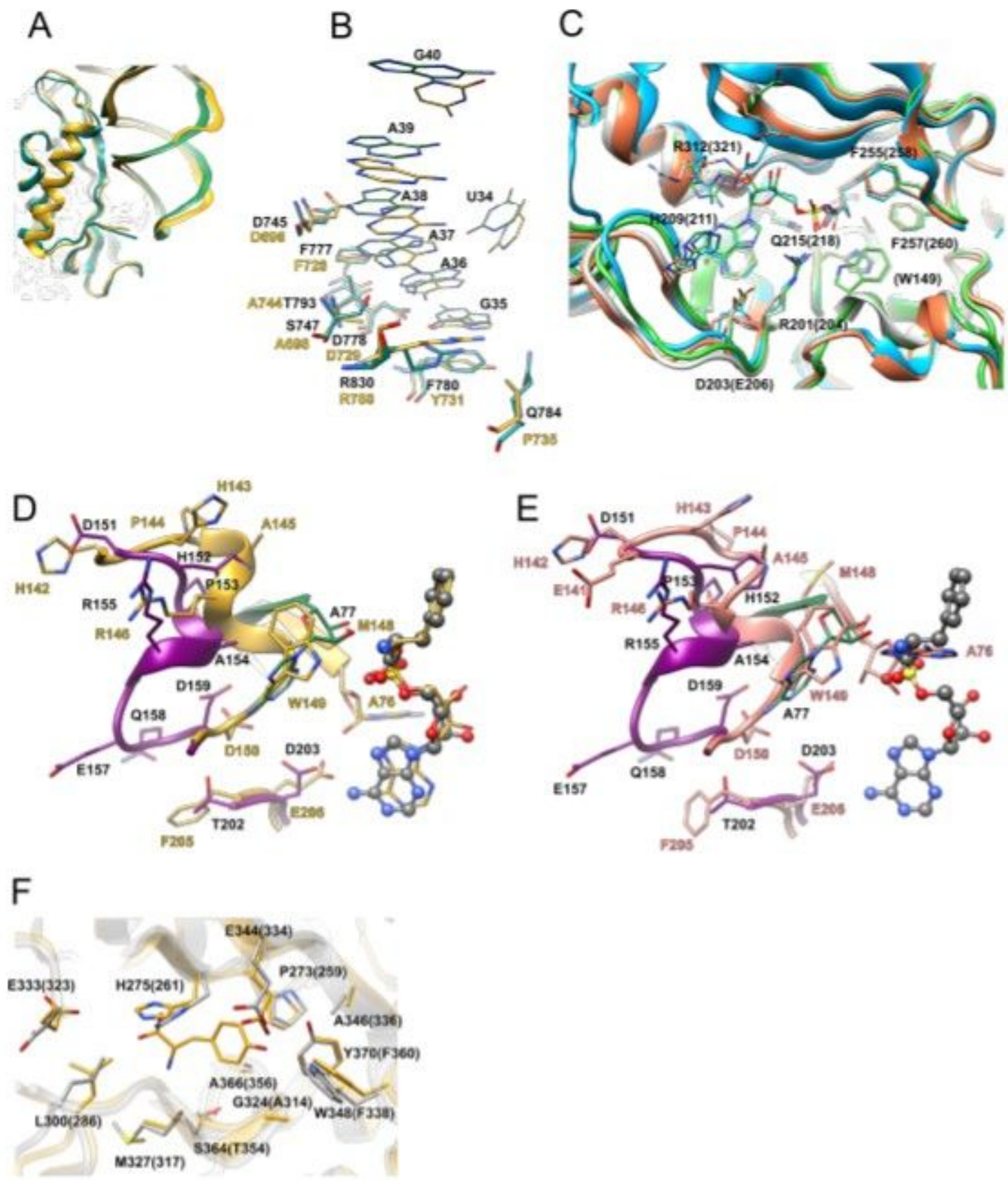

Figure 7

Comparison of MtFRS with T. thermophilus FRS. (A, B) Comparison of the anticodon binding between MtFRSt/F-AMS2 (green, chain C/E) and TtFRSt/adenylate (yellow, PDB 2IY5). In (A) ribbon representation of the b3' domain and anticodon loop. In (B) atomic representation of the anticodon loop and interacting residues. For superposition only domain b3' was used. Labels in black correspond to MtFRS, in yellow to 
TtFRS. (C) Superposition of the aminoacylation domains from MtFRSt/F (coral), MtFRSt/F-AMS1 (blue), TtFRS/F (grey, 1B70), and TtFRS/adenylate (green, 1B7Y). Only select residues are labeled. Parentheses indicate TtFRS. (D) Superposition of the $3^{\prime}$ end binding region in MtFRSt/F-AMS2 and TtFRSt/adenylate. MtFRS is shown in purple (protein), green (tRNA) and grey (F-AMS). TtFRS is shown in yellow. (E) As in (D) but with the TtFRSt complex (pink). Labels in black correspond to MtFRS, in yellow or pink to TtFRS. (F) Superposition of the 27 editing domains (b1s4 subdomain) from MtFRSt/F-AMS2 (grey) and TtFRS/L-Tyr complex (gold, 2AMC).
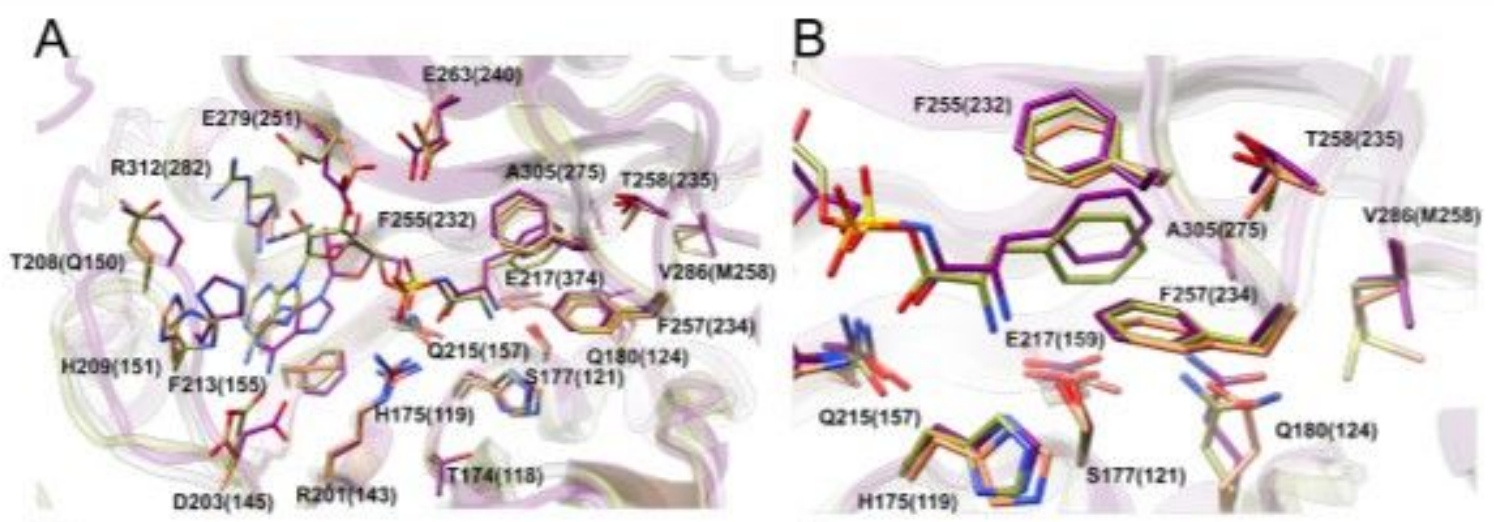

C
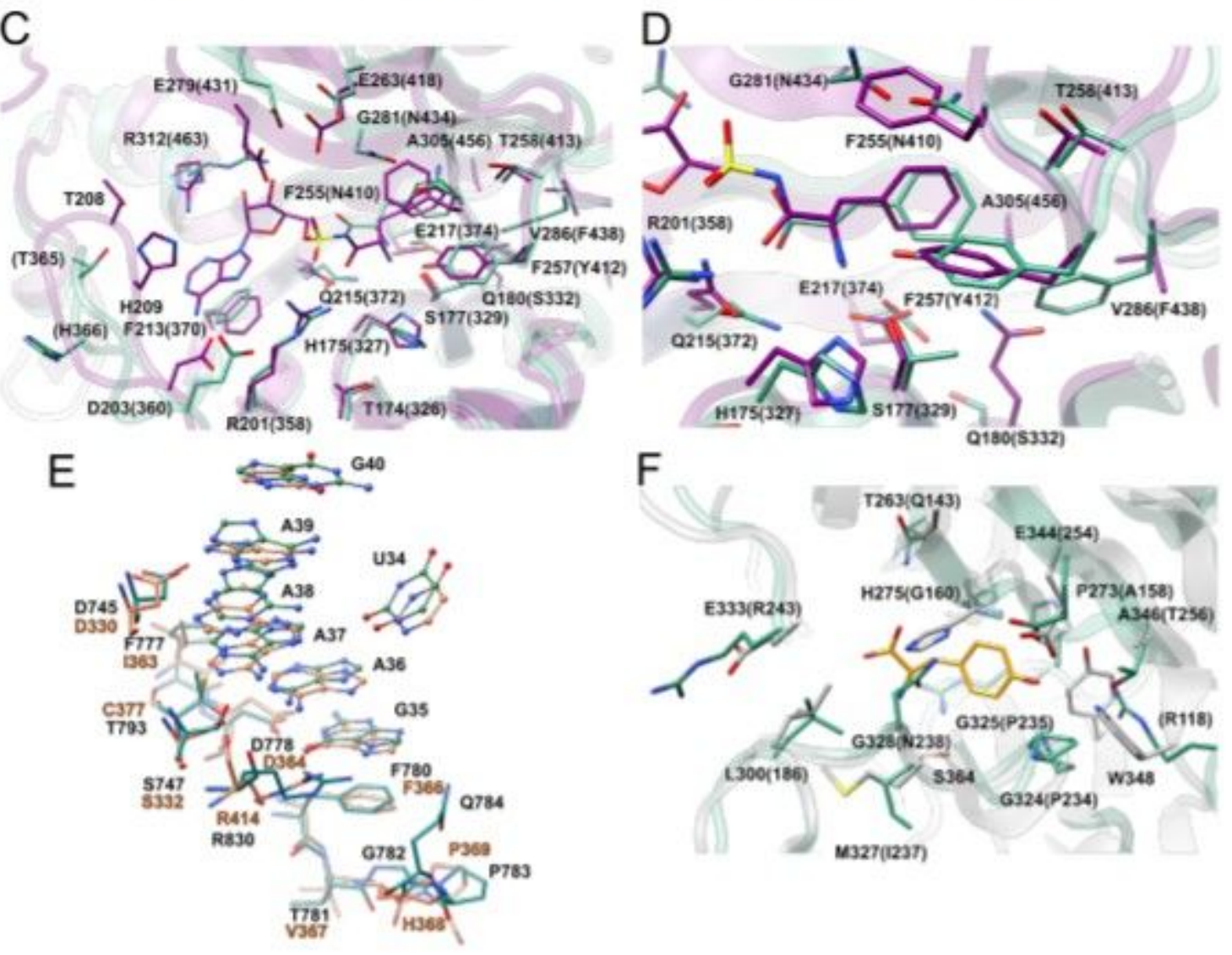

Figure 8 
Comparison of MtFRS with human FRSs. (A-B) Superposition of the aminoacylation domains from MtFRSt/F-AMS2 (purple) with human mitochondrial (hm) enzyme in complex with adenosine-5'[phenylalaninyl-phosphate] (3CMQ, olive green) or with T. termophilus tRNAPhe, hmFRSt (3TUP, coral). In (B) close-up view of the L-Phe subpocket is shown. (C-D). Superposition of the aminoacylation domains from MtFRSt/F-AMS2 (purple) with human cytosolic enzyme (hcFRS) in complex with L-Phe (3L4G, green). In (D) close-up view of the L-Phe subpocket is shown. (E) Superposition of the anticodon binding region of MtFRSt/F-AMS2 (grey) and hmFRSt (coral). Labels in parentheses correspond to the respective human homologs. (F) Superposition of the editing domains (b1s4 subdomain) from MtFRSt/F-AMS2 (grey) and hcFRS (3L4G, green). Gold LTyr from the TtFRS/L-Tyr complex marks the amino acid binding site. 